\title{
Di-Higgs enhancement by neutral scalar as probe of new colored sector
}

\author{
Koji Nakamura $^{1, \mathrm{a}}$, Kenji Nishiwaki ${ }^{2, \mathrm{~b}}$, Kin-ya Oda ${ }^{3, \mathrm{c}}$, Seong Chan Park ${ }^{2,4, \mathrm{~d}}$, Yasuhiro Yamamoto ${ }^{4, \mathrm{e}}$ \\ ${ }^{1}$ IPNS, High Energy Accelerator Research Organization (KEK), Tsukuba, Ibaraki 305-0801, Japan \\ ${ }^{2}$ School of Physics, Korea Institute for Advanced Study (KIAS), Seoul 02455, Republic of Korea \\ ${ }^{3}$ Department of Physics, Osaka University, Osaka 560-0043, Japan \\ ${ }^{4}$ Department of Physics and IPAP, Yonsei University, Seoul 03722, Republic of Korea
}

Received: 2 February 2017 / Accepted: 13 April 2017 / Published online: 2 May 2017

(C) The Author(s) 2017. This article is an open access publication

\begin{abstract}
We study a class of models in which the Higgs pair production is enhanced at hadron colliders by an extra neutral scalar. The scalar particle is produced by the gluon fusion via a loop of new colored particles, and decays into di-Higgs through its mixing with the Standard Model Higgs. Such a colored particle can be the top/bottom partner, such as in the dilaton model, or a colored scalar which can be triplet, sextet, octet, etc., called leptoquark, diquark, coloron, etc., respectively. We examine the experimental constraints from the latest Large Hadron Collider (LHC) data, and discuss the future prospects of the LHC and the Future Circular Collider up to $100 \mathrm{TeV}$. We also point out that the $2.4 \sigma$ excess in the $b \bar{b} \gamma \gamma$ final state reported by the ATLAS experiment can be interpreted as the resonance of the neutral scalar at $300 \mathrm{GeV}$.
\end{abstract}

\section{Introduction}

The di-Higgs production will continue to be one of the most important physics targets in the Large Hadron Collider (LHC) and beyond, since its observation leads to a measurement of the tri-Higgs coupling, and will provide a test if it matches with the Standard Model (SM) prediction [1-11]. Since its production in the SM is destructively interfered with by the top-quark box-diagram contribution, sizable production of di-Higgs directly implies a new physics signature [12].

It is important to examine in which kind of a model the diHiggs signal is enhanced. Indeed the enhancement has been

\footnotetext{
a e-mail: koji.nakamura@cern.ch

b e-mail: nishiken@kias.re.kr

c e-mail: odakin@phys.sci.osaka-u.ac.jp

de-mail: sc.park@yonsei.ac.kr

e e-mail: yamayasu@yonsei.ac.kr
}

pointed out in the models with two Higgs doublets [13-21], type-II seesaw [22], light colored scalars [23], heavy quarks [24], effective operators [25-39], dilaton [40], strongly interacting light Higgs and minimal composite Higgs [41-44], little Higgs [45-47], twin Higgs [48], Higgs portal interactions [40,49-57], supersymmetric partners [5,58-71], and Kaluza-Klein graviton [72]. Other related issues are discussed in Refs. [73-90]. The triple Higgs productions at the LHC and the future circular collider (FCC) are also discussed in Refs. [91-93].

In this paper, we study a class of models in which the di-Higgs process is enhanced by a resonant production of an extra neutral scalar particle. Its production is radiatively induced by the gluon fusion via a loop of new colored particles. Its tree-level decay is due to the mixing with the SM Higgs boson. As concrete examples of the new colored particle that can decay into SM ones in order not to spoil cosmology, we examine the top/bottom partner, such as in the dilaton model, and the colored scalar which are triplet (leptoquark), sextet (diquark), and octet (coloron).

We are also motivated by the anomalous result reported by the ATLAS Collaboration: the $2.4 \sigma$ excess in the search of di-Higgs signal using $b \bar{b}$ and $\gamma \gamma$ final states with the $m_{(b \bar{b})(\gamma \gamma)}\left(=m_{h h}\right)$ invariant mass at around $300 \mathrm{GeV}$ [15]. The excess in $m_{(\gamma \gamma)}$ distribution is right at the SM Higgs mass on top of both the lower and the higher mass-side-band background events. The requested signal cross section roughly corresponds to 90 times larger than what is expected in the $\mathrm{SM}$. Thus the enhancement, if from new physics, should be dramatically generated via e.g. a new resonance at $300 \mathrm{GeV}$.

This paper is organized as follows. In Sect. 2, we present the model. In Sect. 3, we show how the di-Higgs event is enhanced. In Sect. 4, we examine the constraints on the model from the latest results from the ongoing LHC experiment. In Sect. 5, we present a possible explanation for the $2.4 \sigma$ 
excess. In Sect. 6, we summarize our result and provide discussion. In Appendix A, we show how the effective interaction between the new scalar and Higgs is obtained from the original Lagrangian. In Appendix B, we give a parallel discussion for the $Z_{2}$ model. In Appendix $C$, we spell out the possible Yukawa interactions between the colored scalar and the SM fields.

\section{Model}

We consider a class of models in which the di-Higgs $(h h)$ production is enhanced by the schematic diagram depicted in Fig. 1, where $s$ denotes the new neutral scalar and the blob generically represents an effective coupling of $s$ to the pair of gluons via the loop of the extra heavy colored particles. We assume that $h$ and $s$ are lighter and heavier mass eigenstates obtained from the mixing of the neutral component of the $S U(2)_{L}$-doublet $H$ and a real singlet $S$ that couples to the extra colored particles:

$$
\begin{aligned}
& H^{0}=\frac{v+h \cos \theta+s \sin \theta}{\sqrt{2}}, \\
& S=f-h \sin \theta+s \cos \theta,
\end{aligned}
$$

where $\theta$ is the mixing angle and $v$ and $f$ denote the vacuum expectation values (VEVs):

$\left\langle H^{0}\right\rangle=\frac{v}{\sqrt{2}}, \quad\langle S\rangle=f$,

with $v \simeq 246 \mathrm{GeV}$ and $m_{h}=125 \mathrm{GeV}$. We phenomenologically parametrize the effective $s h h$ interaction as

$\Delta \mathcal{L}=-\frac{\mu_{\text {eff }} \sin \theta}{2} s h^{2}$,

where $\mu_{\text {eff }}$ is a real parameter of mass dimension unity, whose explicit form in terms of original Lagrangian parameters is given in Appendix A. We note that the parameter $\mu_{\text {eff }}$ is a

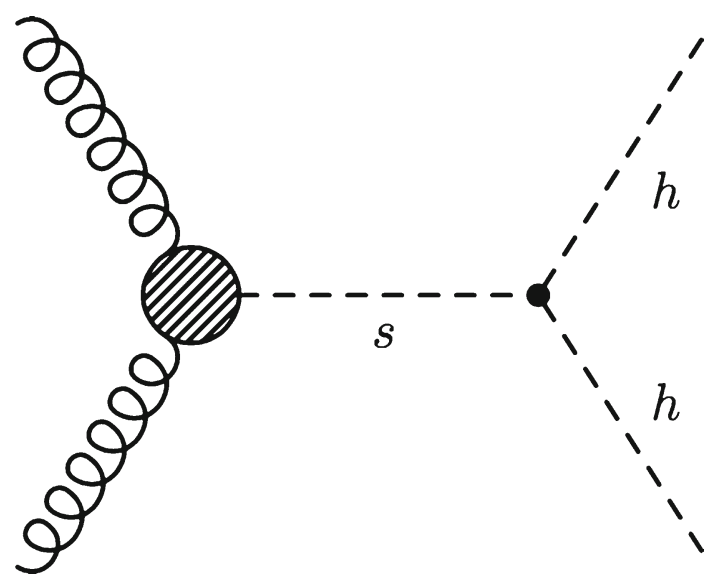

Fig. 1 Di-Higgs $(h h)$ production mediated by $s$
Table 1 Colored particles that may run in the loop represented by the blob in Fig. 1, and their possible parameters. We assume that they are $S U(2)_{L}$ singlets. The electromagnetic charge $Q$ is fixed to allow a mixing with either top or bottom quark for the Dirac spinor and a Yukawa coupling with a pair of SM fermions for the complex scalar;

\begin{tabular}{|c|c|c|c|c|c|c|c|}
\hline \multirow[t]{2}{*}{ Field } & \multicolumn{3}{|c|}{ Dirac spinor } & \multicolumn{4}{|c|}{ Complex scalar } \\
\hline & $T$ & $B$ & $\ldots$ & $\phi_{3}$ & $\phi_{6}$ & $\phi_{8}$ & $\ldots$ \\
\hline$S U(3)_{C}$ & 3 & 3 & $\ldots$ & 3 & 6 & 8 & ... \\
\hline$Q$ & $\frac{2}{3}$ & $-\frac{1}{3}$ & $\ldots$ & $-\frac{1}{3},-\frac{4}{3}$ & $\frac{1}{3},-\frac{2}{3}, \frac{4}{3}$ & $0,-1$ & $\cdots$ \\
\hline$\Delta b_{g}$ & $\frac{2}{3}$ & $\frac{2}{3}$ & $\ldots$ & $\frac{1}{6}$ & $\frac{5}{6}$ & 1 & $\ldots$ \\
\hline$\Delta b_{\gamma}$ & $\frac{16}{9}$ & $\frac{4}{9}$ & $\cdots$ & $\frac{1}{9}, \frac{16}{9}$ & $\frac{2}{9}, \frac{8}{9}, \frac{32}{9}$ & $0, \frac{8}{3}$ & $\cdots$ \\
\hline$\eta$ & & $y_{F} N_{F} \frac{v}{M_{F}}$ & & $\kappa_{\phi} N_{\phi} \frac{f v}{M_{\phi}^{2}}$ & & & \\
\hline
\end{tabular}
see Appendix C. In the last row, $F$ stands for $T$ or $B$

purely phenomenological interface between the experiment and the underlying theory in order to allow a simpler phenomenological expression for the tree-level branching ratios; see Sect. 2.1. We note also that the $\theta$-dependent $\mu_{\text {eff }}(\theta)$ goes to a $\theta$-independent constant in the small mixing limit $\theta^{2} \ll 1$; see Appendix A for detailed discussion. In Sect. 4, it will indeed turn out that only the small, but non-zero, mixing region is allowed in order to be consistent with the signalstrength data of the $125 \mathrm{GeV}$ Higgs at the LHC.

The extra colored particle that runs in the loop, which has been generically represented by the blob in Fig. 1, can be anything that couples to $S$. It should be sufficiently heavy to evade the LHC direct search and decay into SM particles in order not to affect the cosmological evolution. In this paper, we consider the following two possibilities: a Dirac fermion that mixes with either top or bottom quark and a scalar that decays via a new Yukawa interaction with the SM fermions. For simplicity, we assume that the new colored particles are singlet under the $\mathrm{SU}(2)_{L}$ in both cases.

In Table 1, we list the colored particles of our consideration. The higher rank representations of $S U(3)_{C}$ for the colored scalars are terminated at $\mathbf{8}$ in order not to have too higher dimensional Yukawa operators. ${ }^{1}$ The triplet $\phi_{\mathbf{3}}$ is nothing but the leptoquark. It is worth noting that the leptoquark with $Y=-1 / 3$ may account for $R_{D^{(*)}}, R_{K}$, and $(g-2)_{\mu}$ anomalies simultaneously [94].

\subsection{Tree-level decay}

The scalar $s$ may dominantly decay into di-Higgs at the tree level due to the coupling (4):

\footnotetext{
1 The ultraviolet completion of the higher dimensional operator requires other new colored particles. We assume that their contributions are subdominant. E.g. they do not contribute to the effective $g g s$ vertex if they do not have a direct coupling to $S$.
} 

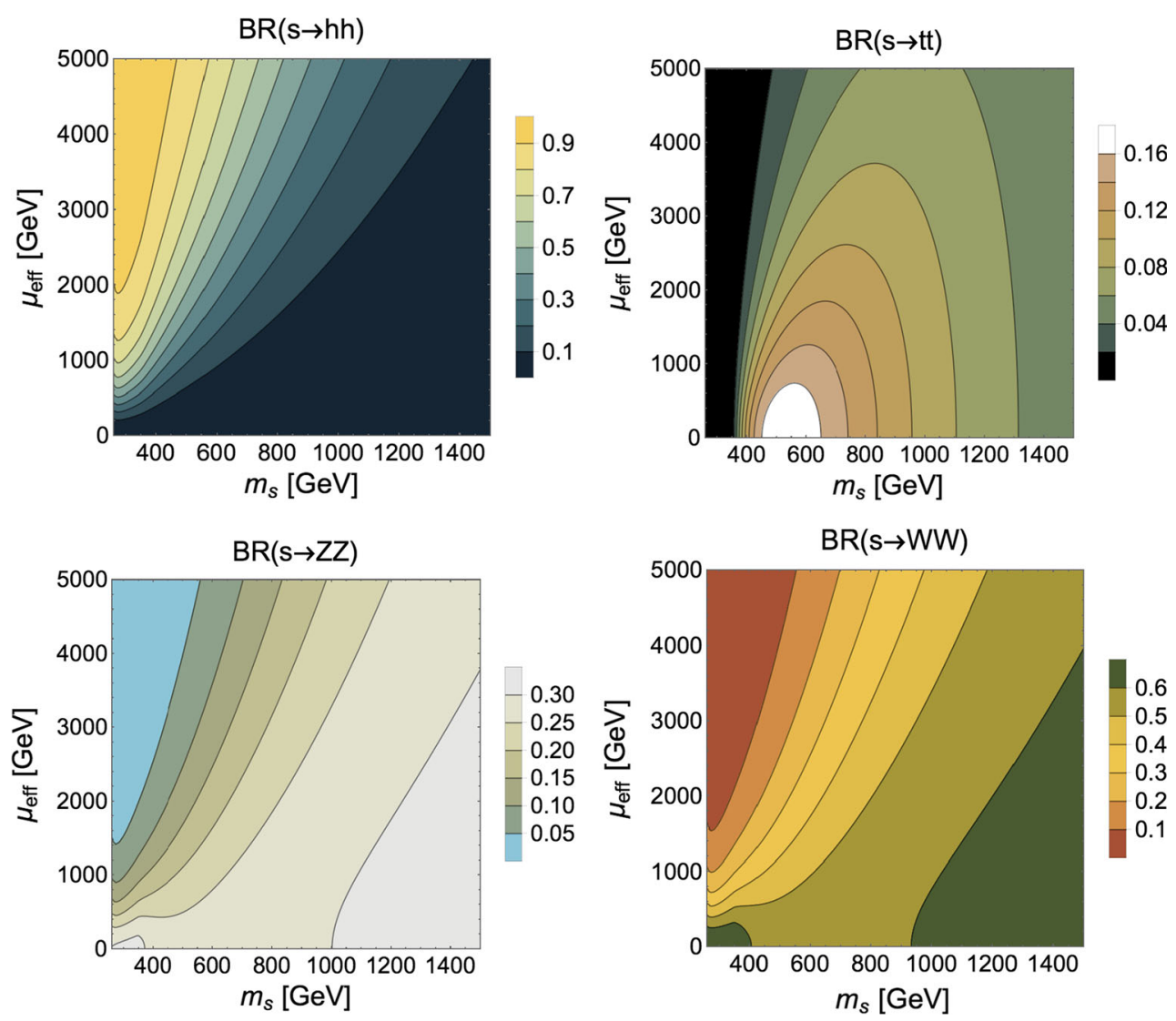

Fig. 2 Tree-level branching ratio for the decay of $s$ in the $\mu_{\text {eff }}$ vs. $m_{s}$ plane

$\Gamma(s \rightarrow h h)=\frac{\mu_{\mathrm{eff}}^{2}}{32 \pi m_{s}} \sqrt{1-\frac{4 m_{h}^{2}}{m_{s}^{2}}} \sin ^{2} \theta$.

For $m_{s}>2 m_{Z}$, the partial decay rate into the pair of vector bosons $s \rightarrow V V$ with $V=W, Z$ are

$\Gamma(s \rightarrow V V)=\frac{m_{s}^{3}}{32 \pi v^{2}} \delta_{V} \sqrt{1-4 x_{V}}\left(1-4 x_{V}+12 x_{V}^{2}\right) \sin ^{2} \theta$,

where $\delta_{Z}=1, \delta_{W}=2$, and $x_{V}=m_{V}^{2} / m_{s}^{2}$; see e.g. Ref. [95]. Similarly for $m_{s}>2 m_{t}$, the partial decay width into a top-quark pair is

$\Gamma(s \rightarrow t \bar{t})=\frac{N_{c} m_{s} m_{t}^{2}}{8 \pi v^{2}}\left(1-\frac{4 m_{t}^{2}}{m_{s}^{2}}\right)^{3 / 2} \sin ^{2} \theta$.

Note that the tree-level branching ratios become independent of $\theta$ thanks to the parametrization (4).

The total decay width $\Gamma_{\text {total }}$ is the sum of the above rates at the tree level. In the small mixing limit $\theta^{2} \ll 1$, the tree-level decay width becomes small and the loop-level decay, which is described in Sect. 2.3, can be comparable to it. The diphoton constraint is severe in this parameter region, as will be discussed in Sect. 4.

In Fig. 2, we plot the tree-level branching ratios in the $\mu_{\text {eff }}$ vs. $m_{s}$ plane. Note that the $\theta$-dependence drops out of the tree-level branching ratios when we use $\mu_{\text {eff }}$ as a phenomenological input parameter as in Eq. (4) because then all the decay channels have the same $\theta$ dependence $\propto \sin ^{2} \theta$.

\subsection{Effective coupling to photons and gluons}

We first consider the vector-like top partner $T$ as the colored particle running in the loop that is represented as the blob in Fig. 1. The bottom partner $B$ can be treated in the same manner, as well as the colored scalars.

The mass of the top partner is given as

$M_{T}=m_{T}+y_{T} f$,

where $m_{T}$ and $y_{T}$ are the vector-like mass of $T$ and the Yukawa coupling between $T$ and $S$, respectively. The top partner $T$ mixes with the SM top quark. We note that limit $m_{T} \rightarrow 0$ corresponds to an effective dilaton model. ${ }^{2}$

Given the kinetic term of gluon that is non-canonically normalized,

2 The particular dilaton model in Ref. [96] corresponds to the identification of the lighter $125 \mathrm{GeV}$ scalar to be an $S$-like one, contrary to this paper. 
$\mathcal{L}_{\text {eff }}=-\frac{1}{4 g_{s}^{2}} G_{\mu \nu}^{a} G^{a \mu \nu}$,

the effective coupling after integrating out the top and $T$ can be obtained by the replacement $\langle S\rangle \rightarrow S$ and $\left\langle H^{0}\right\rangle \rightarrow H^{0}$ in the running coupling; see e.g. Refs. [96,97]:

$$
\begin{aligned}
& \frac{1}{g_{s}^{2}} \longrightarrow \frac{1}{g_{s}^{2}}-\frac{2}{(4 \pi)^{2}}\left(b_{g}^{\text {top }} \frac{h \cos \theta+s \sin \theta}{v}\right. \\
& \left.+\Delta b_{g} y_{T} \frac{-h \sin \theta+s \cos \theta}{M_{T}}\right)
\end{aligned}
$$

where $b_{g}^{\text {top }}$ and $\Delta b_{g}$ are the contributions of top and $T$ to the beta function, respectively. To use this formula, we need to assume the new colored particles are slightly heavier than the neutral scalar. For a Dirac spinor in the fundamental representation, $b_{g}^{\text {top }}=\Delta b_{g}=\frac{1}{2} \times \frac{4}{3}=\frac{2}{3}$. The resultant effective interactions for the canonically normalized gauge fields are

$$
\begin{aligned}
& \mathcal{L}_{\text {eff }}^{h g g}=\frac{\alpha_{s}}{8 \pi v}\left(b_{g}^{\text {top }} \cos \theta-\Delta b_{g} \eta \sin \theta\right) h G_{\mu \nu}^{a} G^{a \mu \nu}, \\
& \mathcal{L}_{\text {eff }}^{s g g}=\frac{\alpha_{s}}{8 \pi v}\left(\Delta b_{g} \eta \cos \theta+b_{g}^{\text {top }} \sin \theta\right) s G_{\mu \nu}^{a} G^{a \mu \nu}, \\
& \mathcal{L}_{\text {eff }}^{h \gamma \gamma}=\frac{\alpha}{8 \pi v}\left(b_{\gamma}^{\mathrm{SM}} \cos \theta-\Delta b_{\gamma} \eta \sin \theta\right) h F_{\mu \nu} F^{\mu \nu}, \\
& \mathcal{L}_{\text {eff }}^{s \gamma \gamma}=\frac{\alpha}{8 \pi v}\left(\Delta b_{\gamma} \eta \cos \theta+b_{\gamma}^{\mathrm{SM}} \sin \theta\right) s F_{\mu \nu} F^{\mu \nu},
\end{aligned}
$$

where $F_{\mu \nu}$ being the (canonically normalized) field strength tensor of the photon, $\alpha_{s}$ and $\alpha$ denoting the chromodynamic and electromagnetic fine structure constants, respectively, $N_{c}=3, b_{\gamma}^{\mathrm{SM}} \simeq-6.5$ and

$\eta=y_{T} N_{T} \frac{v}{M_{T}}$,

with $N_{T}$ being the number of $T$ introduced. The values $\Delta b_{g}=\frac{1}{2} \times \frac{4}{3}=\frac{2}{3}$ and $\Delta b_{\gamma}=N_{c} Q_{T}^{2} \times \frac{4}{3}=\frac{16}{9}$ are listed in Table 1.

The bottom partner $B$ can be treated exactly the same way. According to Table 1, $\Delta b_{\gamma}$ becomes one fourth compared to the above.

For the colored scalar $\phi$, its diagonal mass is given as

$M_{\phi}^{2}=m_{\phi}^{2}+\frac{\kappa_{\phi}}{2}\langle S\rangle^{2}$,

where we have assumed the $Z_{2}$ symmetry $S \rightarrow-S$ for simplicity; $m_{\phi}$ is the original diagonal mass in the Lagrangian; and $\kappa_{\phi}$ is the quartic coupling between $S$ and $\phi .{ }^{3}$ The possible values of the electromagnetic charge of $\phi$ are $Q=-1 / 3$ and $-4 / 3$ for the leptoquark $\phi_{3} ; Q=1 / 3,-2 / 3$, and $4 / 3$ for the color-sextet $\phi_{6}$; and $Q=0$ and -1 for the color-octet $\phi_{\mathbf{8}}$; see Appendix C. Correspondingly the values of $\Delta b_{g}$ are $\frac{1}{2} \times \frac{1}{3}=\frac{1}{6},\left(\frac{N_{c}}{2}+1\right) \times \frac{1}{3}=\frac{5}{6}$, and $N_{c} \times \frac{1}{3}=1$, and $\Delta b_{\gamma}$

\footnotetext{
3 The three point interaction between the neutral and the colored scalar can be introduced. If the sign of the three and the four point couplings are opposite, $\eta$ can be enhanced in some parameter region.
}

are $Q^{2}, 2 Q^{2}$, and $\frac{8}{3} Q^{2}$. Again the effective interactions are obtained as in Eqs. (11)-(14) from the replacement (10) with the substitution $y_{T} / M_{T} \rightarrow \kappa_{\phi} f / M_{\phi}^{2}$, where $f$ has been the VEV of $S$; see Eq. (3). Note that the expression for $\eta$ is now $\eta=\kappa_{\phi} N_{\phi} f v / M_{\phi}^{2}$, where $N_{\phi}$ is the number of $\phi$ introduced. We list all these parameters in Table 1.

\subsection{Loop-level decay}

No direct contact to the gauge bosons are allowed for the singlet scalar $S$, and the tree-level decay of $s$ into a pair of gauge bosons is only via the mixing with the SM Higgs boson. Therefore the decay of $s$ to $g g$ and $\gamma \gamma$ are only radiatively generated. Given the effective operators from the loop of a heavy colored particle,

$\mathcal{L}_{\text {eff }}=-\frac{\alpha_{s} b_{g}}{4 \pi v} s G_{\mu \nu}^{a} G^{a \mu \nu}-\frac{\alpha b_{\gamma}}{4 \pi v} s F_{\mu \nu} F^{\mu \nu}$,

the partial decay widths are

$\Gamma(s \rightarrow g g)=\left(\frac{\alpha_{s} b_{g}}{4 \pi v}\right)^{2} \frac{2 m_{s}^{3}}{\pi}, \quad \Gamma(s \rightarrow \gamma \gamma)=\left(\frac{\alpha b_{\gamma}}{4 \pi v}\right)^{2} \frac{m_{s}^{3}}{4 \pi}$,

where the factor 8 difference comes from the number of degrees of freedom of gluons in the final state. Concretely,

$$
\begin{aligned}
& b_{g}=-\frac{1}{2}\left(\Delta b_{g} \eta \cos \theta+b_{g}^{\mathrm{top}} \sin \theta\right), \\
& b_{\gamma}=-\frac{1}{2}\left(\Delta b_{\gamma} \eta \cos \theta+b_{\gamma}^{\mathrm{SM}} \sin \theta\right) .
\end{aligned}
$$

If we go beyond the scope of this paper and allow the particles in the loop to be charged under $S U(2)_{L}$, then the loop contribution to the decay channels to $Z \gamma, Z Z$ and $W^{+} W^{-}$ might also become significant; see e.g. Ref. [98].

\section{Production of singlet scalar at hadron colliders}

We calculate the production cross section of $s$ via the gluon fusion with the narrow width approximation ${ }^{4}$ :

$$
\begin{aligned}
\hat{\sigma}(g g \rightarrow s) & =\frac{\pi^{2}}{8 m_{s}} \Gamma(s \rightarrow g g) \delta\left(\hat{\sigma}-m_{s}^{2}\right) \\
& =\sigma_{s} m_{s}^{2} \delta\left(\hat{\sigma}-m_{s}^{2}\right),
\end{aligned}
$$

where

$$
\begin{aligned}
\sigma_{s} & :=\frac{\pi^{2}}{8 m_{s}^{3}} \Gamma(s \rightarrow g g)=\left(\frac{\alpha_{s} b_{g}}{4 \pi v}\right)^{2} \frac{\pi}{4} \\
& =36.5 \mathrm{fb} \times\left[\frac{b_{g}}{-1 / 3}\right]^{2}\left[\frac{\alpha_{s}}{0.1}\right]^{2} .
\end{aligned}
$$

\footnotetext{
4 The colored particles running in the blob in Fig. 1 might also have a direct coupling with the quarks in the proton, and possibly change the production cross section of $s$ if it is extremely large. In this paper we assume that this is not the case.
} 


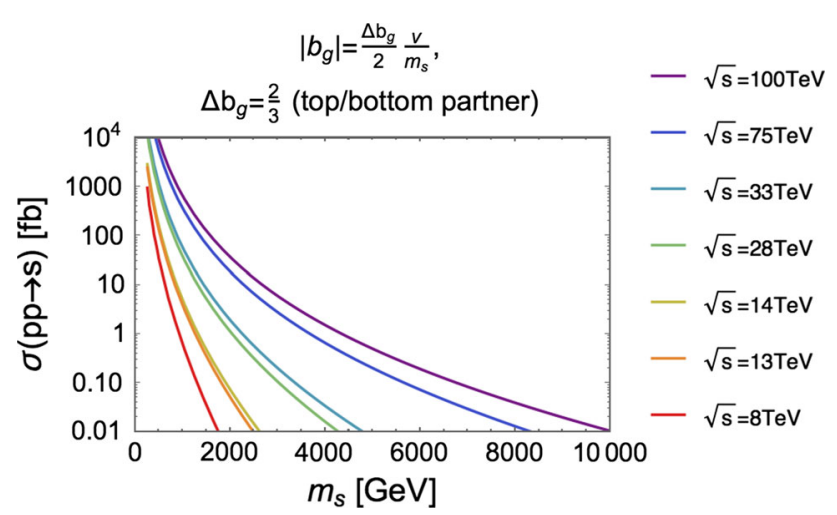

Fig. 3 Production cross section $\sigma(p p \rightarrow s)$ for $\left|b_{g}\right|=\frac{\Delta b_{g}}{2} \frac{v}{m_{s}}$ with $\Delta b_{g}=\frac{2}{3}$ (top/bottom partner). The result for other parameter can be obtained just by a simple scaling $\sigma(p p \rightarrow s) \propto\left(\Delta b_{g}\right)^{2}$; see Eq. (22) with Eq. (19) and Table 1. The $K$-factor is not included in this plot

Therefore, we reach the expression with the gluon parton distribution function (PDF) for the proton $g\left(x, \mu_{F}\right)$ :

$$
\begin{aligned}
& \sigma(p p \rightarrow s)=\sigma_{s} m_{s}^{2} \int_{0}^{1} \mathrm{~d} x_{1} \int_{0}^{1} \mathrm{~d} x_{2} g\left(x_{1}, \mu_{F}\right) g\left(x_{2}, \mu_{F}\right) \\
& \quad \times \delta\left(x_{1} x_{2} s-m_{s}^{2}\right)=\sigma_{s} \tau \frac{\mathrm{d} \mathcal{L}^{g g}}{\mathrm{~d} \tau},
\end{aligned}
$$

where $\tau:=m_{s}^{2} / s$ and

$$
\begin{aligned}
\frac{\mathrm{d} \mathcal{L}^{g g}}{\mathrm{~d} \tau} & =\int_{\tau}^{1} \frac{\mathrm{d} x}{x} g\left(x, \mu_{F}\right) g\left(\tau / x, \mu_{F}\right) \\
& =\int_{\ln \sqrt{\tau}}^{\ln \frac{1}{\sqrt{\tau}}} \mathrm{d} y g\left(\sqrt{\tau} e^{y}, \sqrt{\tau s}\right) g\left(\sqrt{\tau} e^{-y}, \sqrt{\tau s}\right)
\end{aligned}
$$

is the luminosity function, in which the factorization scale $\mu_{F}$ is taken to be $\mu_{F}=\sqrt{\tau s}$. 5

Using the leading order CTEQ6L [99] PDF, we plot in Fig. 3 the production cross section $\sigma(p p \rightarrow s)$ as a function of $m_{s}$ for a phenomenological benchmark setting $\left|b_{g}\right|=$ $\frac{\Delta b_{g}}{2} \frac{v}{m_{s}}$ with $\Delta b_{g}=\frac{2}{3}$ (top/bottom partner). Other particles just scale as $\sigma(p p \rightarrow s) \propto\left(\Delta b_{g}\right)^{2}$. The value $\sqrt{s}=14 \mathrm{TeV}$ is motivated by the High-Luminosity LHC; 28 and $33 \mathrm{TeV}$ by the High-Energy LHC (HE-LHC); and 75, and $100 \mathrm{TeV}$ by the Future Circular Collider (FCC) [100-102].

We see that typically the top/bottom partner models give a cross section $\sigma(p p \rightarrow s) \gtrsim 1 \mathrm{fb}$, which could be accessed by a luminosity of $\mathcal{O}\left(\mathrm{ab}^{-1}\right)$, for the scalar mass $m_{s} \lesssim 1.3$, 2, and $4 \mathrm{TeV}$ at the LHC, HE-LHC, and FCC, respectively.

Several comments are in order:

- Our setting corresponds to putting $M_{T}=y_{T} N_{T} m_{s}$ in Eq. (15) in order to reflect the naive scaling of $\eta \sim v / f$ with $f \sim m_{s}$; recall that we need $M_{T} \gtrsim m_{s}$ to justify

\footnotetext{
5 Notational abuse of $s$ for the singlet scalar field and for the Mandelstam variable of $p p$ scattering should be understood.
}

integrating out the top partner to write down the effective interactions (11)-(14).

- Here we have used the leading order parton distribution function. The higher order corrections may be approximated by multiplying an overall factor $K$, the so-called $K$-factor, which takes value $K \simeq 1.6$ for the SM Higgs production at LHC; see e.g. Ref. [95].

- The SM cross section for $p p \rightarrow h h$ is of the order of $10 \mathrm{fb}$ and $10^{3} \mathrm{fb}$ for $\sqrt{s}=8 \mathrm{TeV}$ and $100 \mathrm{TeV}$, respectively [12]. We are interested in the on-shell production of $s$, and the non-resonant SM background can be discriminated by kinematical cuts. The detailed study is beyond the scope of this paper and will be presented elsewhere.

- When we consider the new resonance with a narrow width (21), we can neglect the box contribution from the extra colored particles as the box contribution gets a suppression factor ${ }^{6}$

$$
\frac{\mu_{\mathrm{eff}} M_{T}}{32 \pi v^{2}} \sin ^{3} \theta \sim 10^{-4}\left[\frac{\mu_{\mathrm{eff}}}{1 \mathrm{TeV}}\right]\left[\frac{M_{T}}{1 \mathrm{TeV}}\right]\left[\frac{\sin \theta}{0.1}\right]^{3} \ll 1 .
$$

6 In the SM, the $g g \rightarrow h h$ cross section takes the following form at the leading order [12]:

$$
\begin{aligned}
& \hat{\sigma}_{\mathrm{LO}}^{\mathrm{SM}}(g g \rightarrow h h)=\int_{\hat{t}_{-}}^{\hat{t}_{+}} \mathrm{d} \hat{t} \frac{G_{\mathrm{F}}^{2} \alpha_{s}^{2}}{256(2 \pi)^{3}} \\
& \times\left[\left|\frac{\mu_{h h h} v}{\left(\hat{s}-m_{h}^{2}\right)+i m_{h} \Gamma_{h}} F_{\triangle}^{\mathrm{SM}}+F_{\square}^{\mathrm{SM}}\right|^{2}+\left|G_{\square}^{\mathrm{SM}}\right|^{2}\right],
\end{aligned}
$$

where $G_{\mathrm{F}}$ is the Fermi constant; $\mu_{h h h}=3 m_{h}^{2} / v$ is the $h h h$ coupling in the SM; and $F_{\triangle}^{\mathrm{SM}}, F_{\square}^{\mathrm{SM}}$, and $G_{\square}^{\mathrm{SM}}$ are the triangular and box form factors, approaching $F_{\triangle}^{\mathrm{SM}} \rightarrow 2 / 3, F_{\square}^{\mathrm{SM}} \rightarrow-2 / 3$, and $G_{\square}^{\mathrm{SM}} \rightarrow 0$ in the large top-quark-mass limit. A large cancellation takes place between $F_{\triangle}^{\mathrm{SM}}$ and $F_{\square}^{\mathrm{SM}}$ as is well known.

For the on-shell resonance production of $s$, on the other hand, the triangle contribution from the fermion loops dominates over the box loop contribution: The new triangle contribution for $s$ can be well approximated by replacing the expression for the $\mathrm{SM}$ as

$$
\begin{gathered}
\mu_{h h h} \rightarrow \mu_{\mathrm{eff}} \sin \theta, \quad m_{h} \rightarrow m_{s}, \quad \Gamma_{h} \rightarrow \Gamma_{s}, \\
F_{\Delta}^{\mathrm{SM}} \rightarrow \Delta b_{g} \eta \cos \theta+b_{g}^{\text {top }} \sin \theta,
\end{gathered}
$$

and the new box contribution of the top partner can be obtained from that of the SM top quark with the multiplicative factor

$$
\frac{N_{T} y_{T}^{2} \sin ^{2} \theta}{y_{t}^{2} / 2} \frac{y_{T}^{2} f^{2}}{M_{T}^{2}}
$$

Finally, taking the ratio of the size of the box contribution and the triangle contribution with $\Delta b_{g}=2 / 3$ and $\eta=y_{T} N_{T} v / M_{T} \sim N_{T} v / M_{T}$, $y_{T} \sim y_{t}$, and $m_{s} \Gamma_{s} \sim \mu_{\text {eff }}^{2} \sin ^{2} \theta / 32 \pi$, we get the result in Eq. (25). 


\section{LHC constraints}

We examine LHC constraints on the model for various $m_{s}$. That is, we verify constraints from $125 \mathrm{GeV}$ Higgs signal strength, from $s \rightarrow Z Z \rightarrow 4 l$ search, from $s \rightarrow \gamma \gamma$ search, and from the direct search of the colored particles running in the blob in Fig. 1.

\subsection{Bound from Higgs signal strength}

We first examine the bound on $\theta$ and $\eta$ from the Higgs signal strengths in various channels. The "partial signal strength" for the Higgs production becomes

$$
\begin{gathered}
\mu_{\mathrm{ggF}}=\left(\cos \theta-\frac{\Delta b_{g}}{b_{g}^{\mathrm{top}}} \eta \sin \theta\right)^{2}, \\
\mu_{\mathrm{VBF}}=\mu_{\mathrm{VH}}=\mu_{\mathrm{ttH}}=\cos ^{2} \theta,
\end{gathered}
$$

where $\mathrm{ggF}, \mathrm{VBF}, \mathrm{VH}$, and $\mathrm{ttH}$ are the gluon fusion, vectorboson fusion, associated production with vector, and that with a pair of top quarks, respectively; see e.g. Ref. [103] for details. Similarly, the partial signal strength for the Higgs decay is

$$
\begin{aligned}
& \mu_{h \rightarrow \gamma \gamma}=\left(\cos \theta-\frac{\Delta b_{\gamma}}{b_{\gamma}^{\mathrm{SM}}} \eta \sin \theta\right)^{2}\left(\frac{\Gamma_{h}}{\Gamma_{h}^{\mathrm{SM}}}\right)^{-1}, \\
& \mu_{h \rightarrow g g}=\mu_{\mathrm{ggF}}\left(\frac{\Gamma_{h}}{\Gamma_{h}^{\mathrm{SM}}}\right)^{-1}, \\
& \mu_{h \rightarrow f \bar{f}, W W, Z Z}=\cos ^{2} \theta\left(\frac{\Gamma_{h}}{\Gamma_{h}^{\mathrm{SM}}}\right)^{-1},
\end{aligned}
$$

where the ratio of the total widths is given by

$$
\begin{aligned}
& \left(\frac{\Gamma_{h}}{\Gamma_{h}^{\mathrm{SM}}}\right)=\mathrm{Br}_{h \rightarrow \mathrm{SM} \text { others }}^{\mathrm{SM}} \cos ^{2} \theta \\
& +\mathrm{Br}_{h \rightarrow \gamma \gamma}^{\mathrm{SM}}\left(\cos \theta-\frac{\Delta b_{\gamma}}{b_{\gamma}^{\mathrm{SM}} \eta \sin \theta}\right)^{2}+\mathrm{Br}_{h \rightarrow g g}^{\mathrm{SM}} \mu_{\mathrm{ggF}},
\end{aligned}
$$

with $\mathrm{Br}_{h \rightarrow \text { SM others }}^{\mathrm{SM}}=0.913, \mathrm{Br}_{h \rightarrow \gamma \gamma}^{\mathrm{SM}}=0.002$ and $\mathrm{Br}_{h \rightarrow g g}^{\mathrm{SM}}=0.085$. We compare these values with the corresponding constraints given in Ref. [103]. Results are shown in Fig. 4 for the matter contents summarized in Table 1. We note that the region near $\theta \simeq 0$ is always allowed by the signal-strength constraints, though it is excluded by the diphoton search as we will see.

\subsection{Bound from $s \rightarrow Z Z \rightarrow 4 l$}

One of the strongest constraints on the model comes from the heavy Higgs search in the four lepton final state at $\sqrt{s}=13 \mathrm{TeV}$ at ATLAS [104]. Experimentally, an upper bound is put on the cross section $\sigma(p p \rightarrow s \rightarrow Z Z \rightarrow 4 l)$, with $l=e, \mu$, for each $m_{s}$. Its theoretical cross section is obtained by multiplying the production cross section (23) by the branching ratio $\mathrm{BR}(s \rightarrow Z Z)=\Gamma(s \rightarrow Z Z) / \Gamma(s \rightarrow$ all $)$ and $\left(\mathrm{BR}_{\mathrm{SM}}(Z \rightarrow e e, \mu \mu)\right)^{2} \simeq(6.73 \%)^{2}$; see Sect. 2.1 .

In Fig. 6 , we plot $2 \sigma$ excluded regions on the $\mu_{\text {eff }}$ vs. $m_{s}$ plane with varying $b_{g}$ from 0 to 1 with incrementation 0.2 . The weakest bound starts to exist on the plane from $b_{g}=0.2$. The $K$-factor is set to be $K=1.6$. The experimental bound becomes milder for large $\mu_{\text {eff }}$ because the di-Higgs channel dominate the decay of the neutral scalar. The large fluctuation of the bound is due to the statistical fluctuation of the original experimental constraint.

We note that, though we have focused on the strongest constraint at the low $m_{s}$ region, the other decay channels of $W W \rightarrow l v q q$ and of $Z Z \rightarrow \nu v q q$ and $l l v v$ may also become significant at the high mass region $m_{s} \gtrsim 700 \mathrm{GeV}$.

\subsection{Bound from $s \rightarrow \gamma \gamma$}

A strong constraint comes from the heavy Higgs search in the di-photon final state at $\sqrt{s}=13 \mathrm{TeV}$ at ATLAS [105]. Experimentally, an upper bound is put on the cross section $\sigma(p p \rightarrow s \rightarrow \gamma \gamma)$ for each $m_{s}$. Its theoretical cross section is obtained by multiplying the production cross section (23) by the branching ratio $\mathrm{BR}(s \rightarrow \gamma \gamma)=\Gamma(s \rightarrow \gamma \gamma) / \Gamma(s \rightarrow$ all); see Sect. 2.1. Since this constraint is strong in the small mixing region, where the loop-level decay is comparable to the tree-level decay, we include the loop-level decay channels into $\Gamma(s \rightarrow$ all $)$ for this analysis; see Sect. 2.3.

In Fig 7, we plot the $2 \sigma$-excluded regions on $\mu_{\text {eff }}$ vs. $m_{s}$ plane for $\sin \theta=0.01,0.03,0.05$, and 0.1 , with varying $b_{g} b_{\gamma}$ from 0 to 2 with incrementation 0.2 . $K$-factor is set to be $K=$ 1.6. If $\sin \theta=0.01$, broad region is excluded for $b_{g} b_{\gamma}=0.4$. On the other hand, the experimental bound is negligibly weak in the case of $\sin \theta=0.1$. The large fluctuation of the bound is due to the statistical fluctuation of the original experimental constraint.

In Fig. 8 , we plot the same $2 \sigma$-excluded regions on the $\sin \theta$ vs. $\eta$ plane for $m_{s}=300,600,900,1200$, and $1500 \mathrm{GeV}$. In the left and right panels, we set $\mu_{\mathrm{eff}}=1 \mathrm{TeV}$ and $\mu_{\mathrm{eff}}=\sqrt{3} m_{s}^{2} / v$. The latter corresponds to $\Gamma(s \rightarrow$ $h h)=\sum_{V=W, Z} \Gamma(s \rightarrow V V)$ which is chosen such that there are sizable di-Higgs event and that $\mu_{\text {eff }}$ is not too large. $K$-factor is set to be $K=1.6$. We emphasize that the small mixing limit $\sin \theta \rightarrow 0$ is always excluded by the di-photon channel in contrast to the other bounds, though it cannot be seen in Fig. 8 in the small $\eta$ region due to the resolution.

The bound from $s \rightarrow Z \gamma$ is weaker and we do not present the result here. 

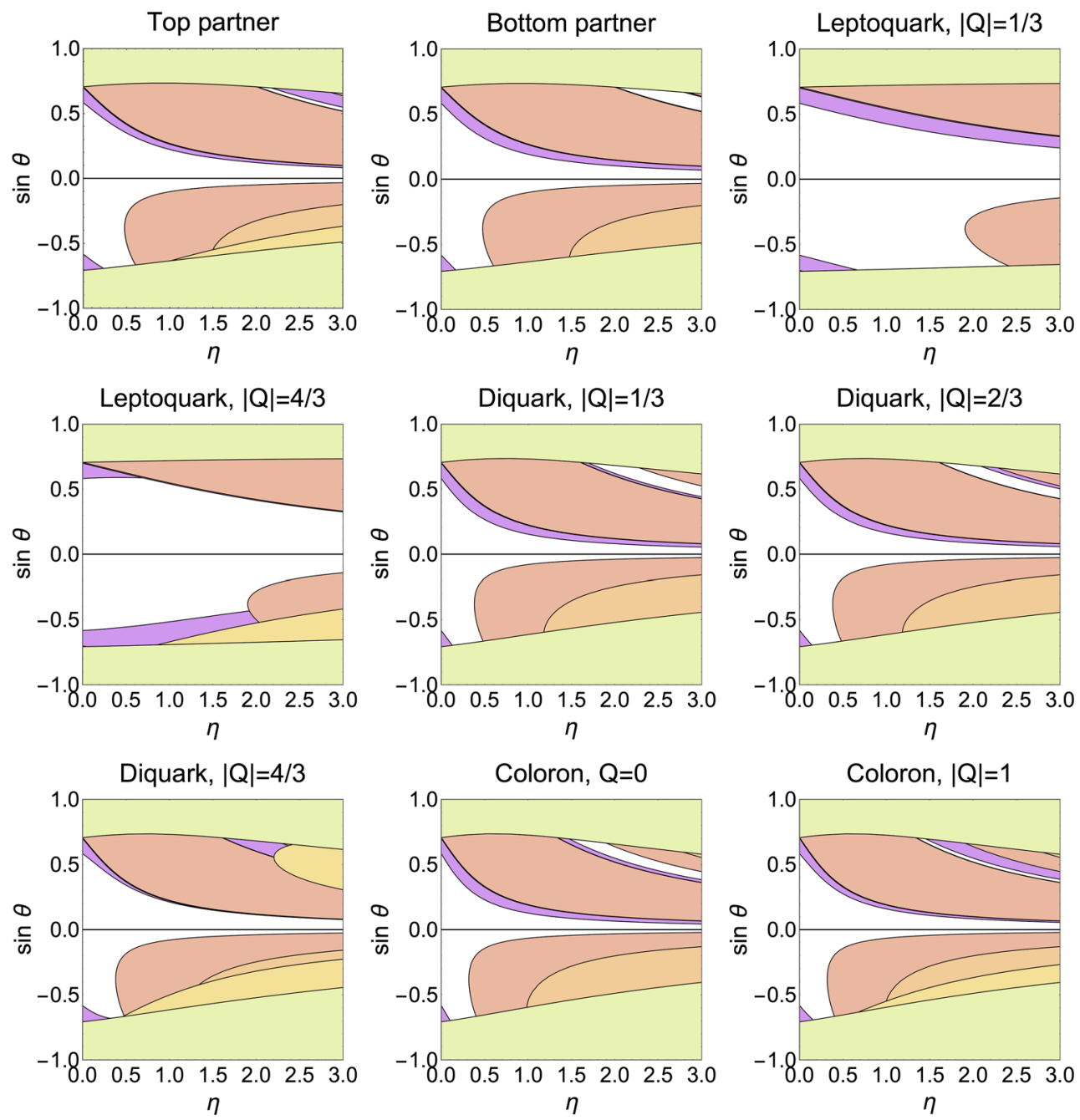

Fig. $42 \sigma$-excluded regions from the signal strength of $125 \mathrm{GeV}$ Higgs are shaded. The color represents the contribution from each channel; see Fig. 5 for details
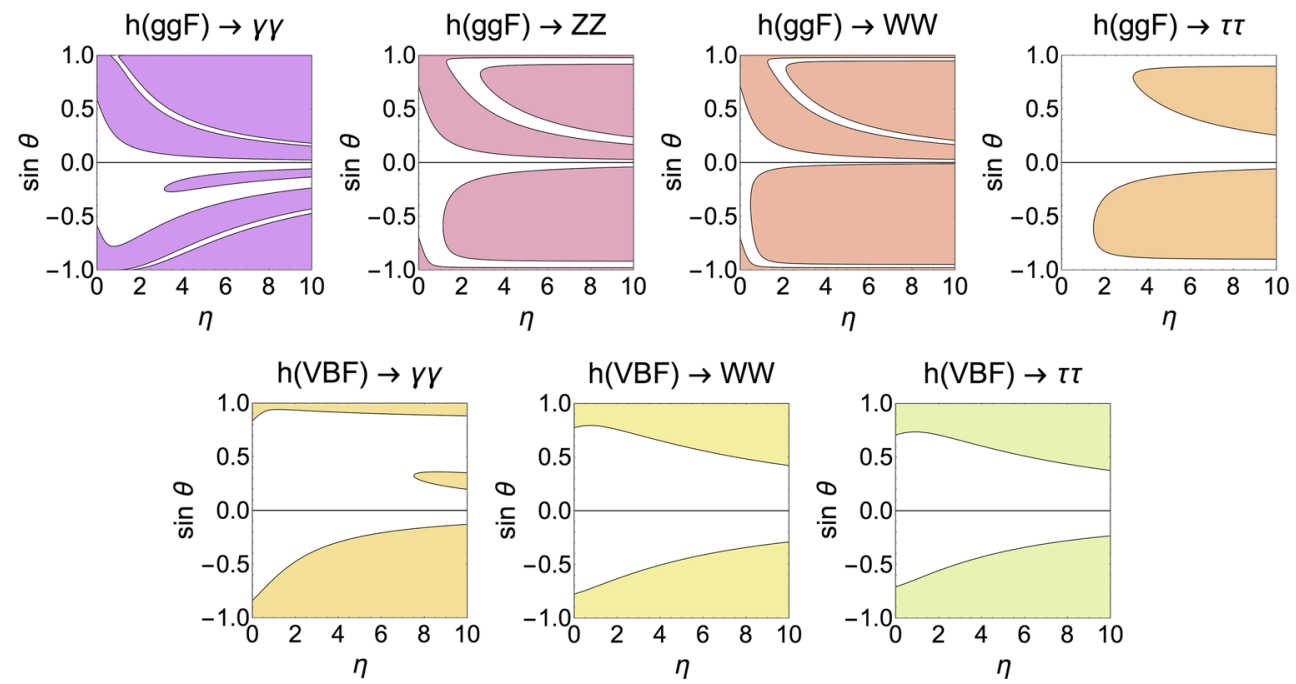

Fig. 5 The $2 \sigma$-excluded regions from the signal strength of $125 \mathrm{GeV}$ Higgs. The top-partner parameters are chosen as an illustration to present the contribution from each channel 


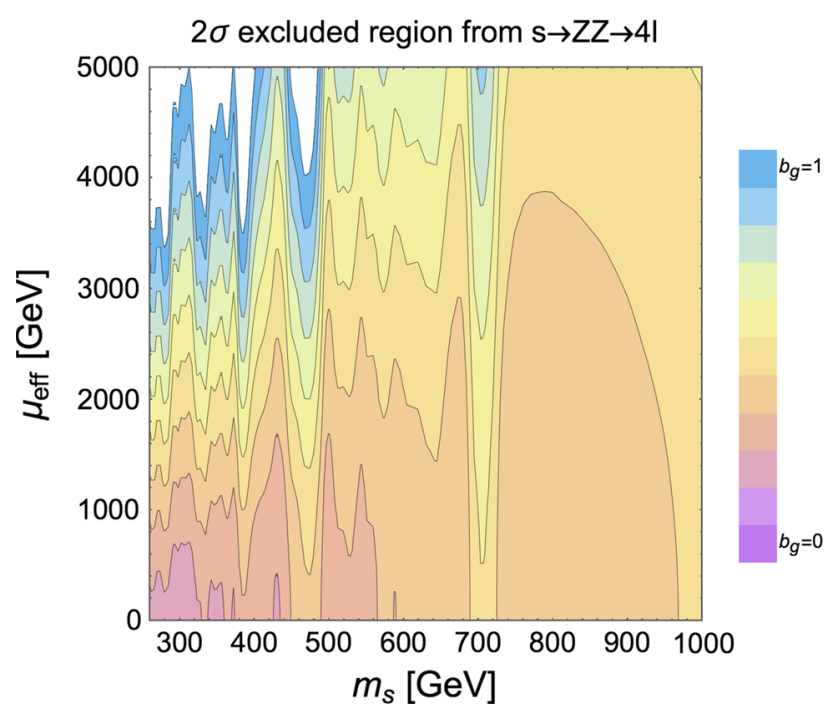

Fig. 6 The $2 \sigma$-excluded regions from $s \rightarrow Z Z \rightarrow 4 l$ bound in the $\mu_{\text {eff }}$ vs. $m_{s}$ plane. The color is changed in increments of 0.1 . The weakest bound starts existing from $b_{g}=0.2$. $K$-factor is set to be $K=1.6$

\subsection{Bound from direct search for colored particles}

We first review the mass bound on the extra colored particles. For the $S U(2)_{L}$ singlet $T$ and $B[106,107]$,

$M_{T}, M_{B} \gtrsim 800 \mathrm{GeV}$.

The mass bound for the leptoquark $\phi_{\mathbf{3}}$, diquark $\phi_{\mathbf{6}}$, and coloron $\phi_{\mathbf{8}}$ are given in Refs. [108-111] as

$m_{\phi_{\mathbf{3}}} \gtrsim 0.7-1.1 \mathrm{TeV}, \quad m_{\phi_{\mathbf{6}}} \gtrsim 7 \mathrm{TeV}, \quad m_{\phi_{\mathbf{8}}} \gtrsim 5.5 \mathrm{TeV}$,

respectively, depending on the possible decay channels.

For the top partner $M_{T} \gtrsim 800 \mathrm{GeV}$ with $\theta \simeq 0$, we get $\eta \lesssim 0.3 y_{T} N_{T}$. Therefore, we need rather large Yukawa coupling $y_{T} \simeq 2.2$ for $N_{T}=1$ in order to account for Eq. (33) by Eq. (35). ${ }^{7}$ The same argument applies for the bottom partner since it has the same $\Delta b_{g}=2 / 3$.

Similarly for a colored scalar with $M_{\phi} \gtrsim 0.7,1.1,5.5$, and $7 \mathrm{TeV}$, we get $\eta \lesssim \kappa_{\phi} N_{\phi} \frac{f}{2 \mathrm{TeV}}, \kappa_{\phi} N_{\phi} \frac{f}{4.9 \mathrm{TeV}}, \kappa_{\phi} N_{\phi} \frac{f}{123 \mathrm{TeV}}$, and $\kappa_{\phi} N_{\phi} \frac{f}{200 \mathrm{TeV}}$, respectively. For $\theta \simeq 0$, the value of $b_{g}$ is suppressed or enhanced by extra factors $\frac{1}{6} / \frac{2}{3}=1 / 4$, $\frac{5}{6} / \frac{2}{3}=\frac{5}{4}$, and $1 / \frac{2}{3}=3 / 2$, respectively, compared to the top partner. Therefore, from Eq. (36), we need $\kappa_{\phi} N_{\phi} f \gtrsim 5-$ $13 \mathrm{TeV}, 106 \mathrm{TeV}$, and $54 \mathrm{TeV}$ for $\phi_{\mathbf{3}}, \phi_{\mathbf{6}}$, and $\phi_{\mathbf{8}}$, respectively, in order to account for the $2.4 \sigma$ excess at $\theta^{2} \ll 1$.

\footnotetext{
7 Strictly speaking, the bound on $M_{T}$ slightly changes when $N_{T} \geq 2$, and hence the bound for $y_{T} N_{T}$ could be modified accordingly.
}

5 Accounting for $2.4 \sigma$ excess of $b \bar{b} \gamma \gamma$ by $m_{s}=300 \mathrm{GeV}$

It has been reported by the ATLAS Collaboration that there exists $2.4 \sigma$ excess of $h h$-like events in the $b \bar{b} \gamma \gamma$ final state [15]. This corresponds to the extra contribution to the SM cross section $^{8}$

$\sigma(p p \rightarrow h h)_{\mathrm{extra}, 8 \mathrm{TeV}} \simeq 0.8 \mathrm{pb}$

In Fig. 9, we plot the branching ratio at $m_{h}=300 \mathrm{GeV}$ as a function of $\mu_{\text {eff. }}$.

\subsection{Signal}

With $m_{s}=300 \mathrm{GeV}$, we get the luminosity functions

$$
\left.\tau \frac{\mathrm{d} \mathcal{L}^{g g}}{\mathrm{~d} \tau}\right|_{m_{s}=300 \mathrm{GeV}} \simeq \begin{cases}17.2 & (\sqrt{s}=8 \mathrm{TeV}) \\ 54.5(64.2) & (\sqrt{s}=13(14) \mathrm{TeV}) \\ 263(357) & (\sqrt{s}=28(33) \mathrm{TeV}) \\ 2310(1470) & (\sqrt{s}=100(75) \mathrm{TeV})\end{cases}
$$

That is,

$$
\begin{aligned}
& \sigma(p p \rightarrow s)_{m_{s}=300 \mathrm{GeV}} \simeq\left[\frac{b_{g}}{-1 / 3}\right]^{2}\left[\frac{\alpha_{s}}{0.1}\right]^{2}\left[\frac{K}{1.6}\right] \\
& \quad \times \begin{cases}1.0 \mathrm{pb} & (\sqrt{s}=8 \mathrm{TeV}), \\
3.2(3.8) \mathrm{pb} & (\sqrt{s}=13(14) \mathrm{TeV}), \\
15(18) \mathrm{pb} & (\sqrt{s}=28(33) \mathrm{TeV}), \\
130(83) \mathrm{pb} & (\sqrt{s}=100(75) \mathrm{TeV}) .\end{cases}
\end{aligned}
$$

In Fig. 10, we plot the preferred contour to explain the $2.4 \sigma$ excess at $m_{s}=300 \mathrm{GeV}$, where the shaded region is excluded at the $95 \% \mathrm{CL}$ by the $\sigma(p p \rightarrow s \rightarrow Z Z \rightarrow$ $4 l)_{13 \mathrm{TeV}}$ constraint that has been discussed in Sect. 4.2. We have assumed the $K$-factor $K=1.6$.

We see that at the benchmark point $\theta \simeq 0$, the lowest and highest possible values of $\mu_{\mathrm{eff}}$ and $\eta$ are, respectively,

$\mu_{\mathrm{eff}} \gtrsim 800 \mathrm{GeV}, \quad \eta \lesssim \begin{cases}0.66 & \text { top/bottom partner, } \\ 2.6 & \text { leptoquark, } \\ 0.53 & \text { diquark, } \\ 0.44 & \text { coloron, }\end{cases}$

in order to account for the cross section (33). The ratio of the upper bound on $\eta$ is given by the scaling $\propto\left(\Delta b_{g}\right)^{2}$.

\footnotetext{
8 At $\sqrt{s}=8 \mathrm{TeV}, \sigma_{\mathrm{SM}}(p p \rightarrow h h)=9.2 \mathrm{fb}$. The expected number of events are $1.3 \pm 0.5,0.17 \pm 0.04$, and 0.04 for the non- $h$ background, single $h$, and the SM $h h$ events, respectively. Since the observed number of events is 5 , the excess is $5-1.3-0.17=3.5$, which is $3.5 / 0.04=87.5$ times larger than the SM $h h$ events. Therefore, the excess corresponds to $9.2 \mathrm{fb} \times 87.5=0.8 \mathrm{pb}$.
} 

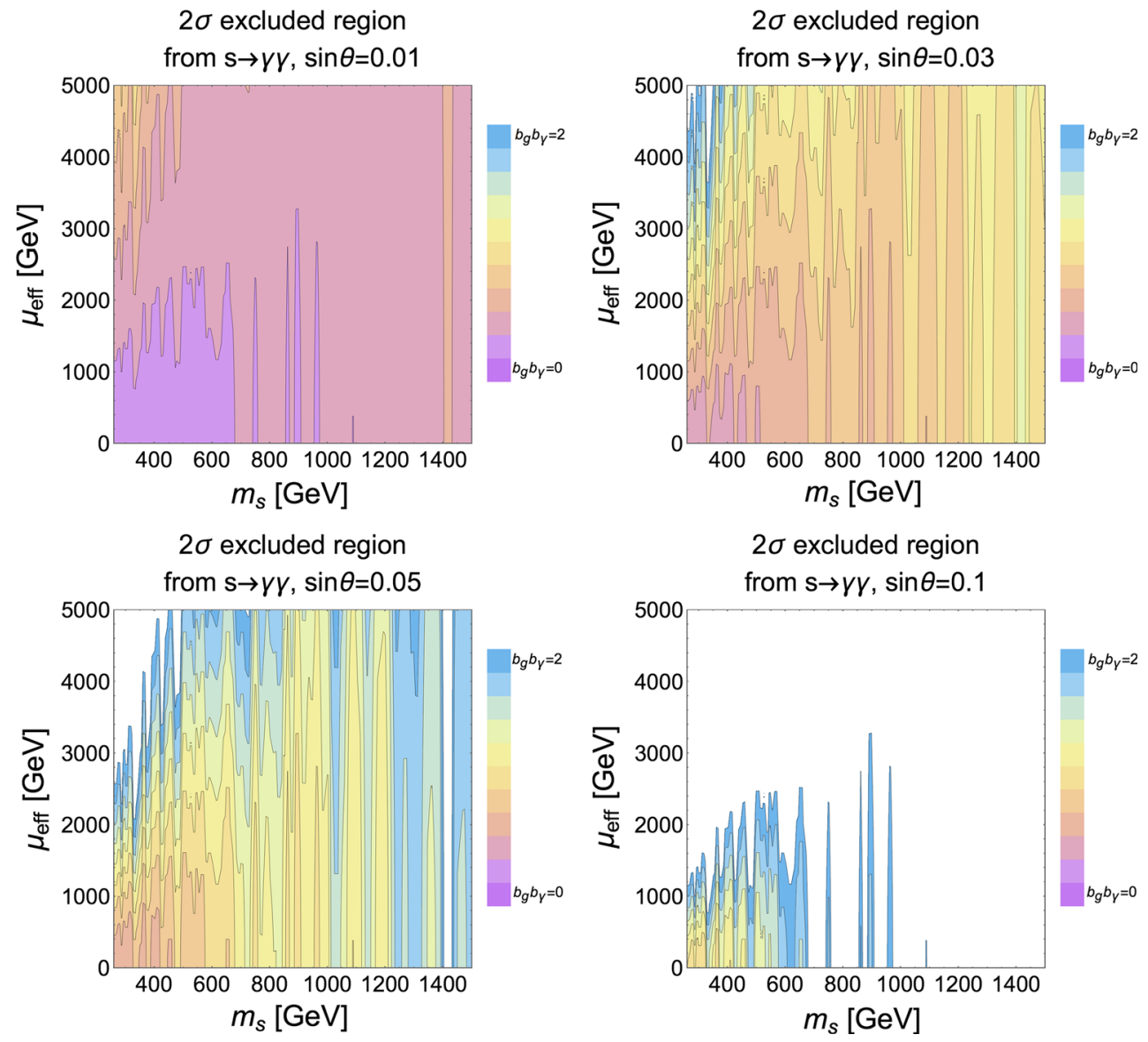

Fig. 7 The $2 \sigma$-excluded regions from $s \rightarrow \gamma \gamma$ bound in the $\mu_{\text {eff }}$ vs. $m_{s}$ plane for various $\sin \theta$. The color is changed in increments of 0.2 . $K$-factor is set to be $K=1.6$
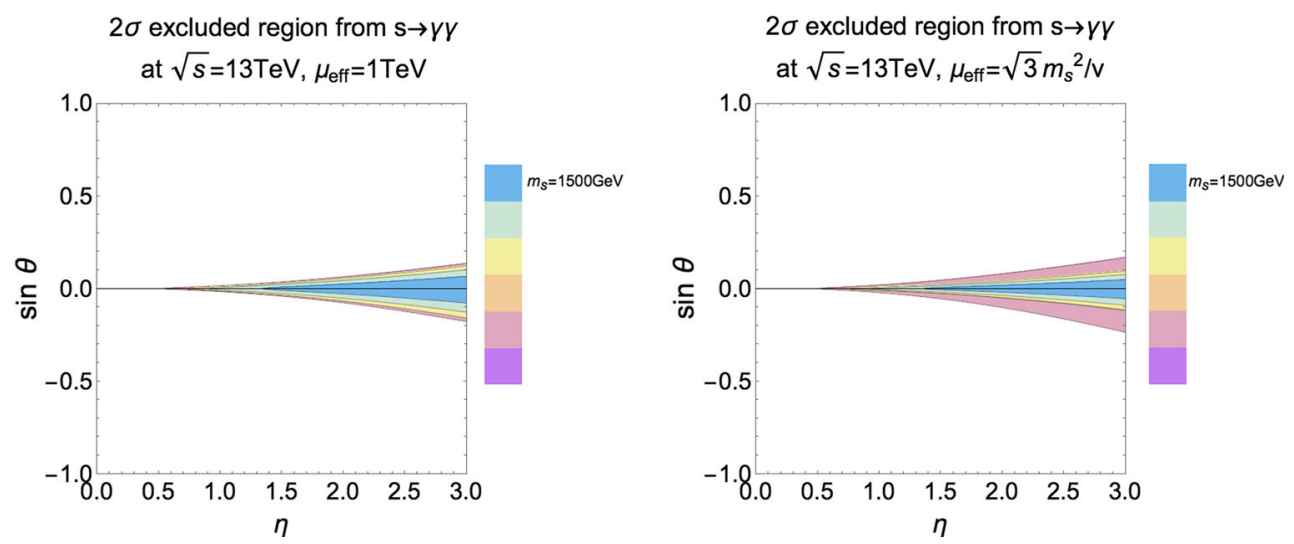

Fig. 8 The $2 \sigma$-excluded regions from $s \rightarrow \gamma \gamma$ bound in the $\sin \theta$ vs. $\eta$ plane for various $m_{s}$ with $\mu_{\text {eff }}=1$ TeV and $\sqrt{3} m_{s}^{2} / v$. The color is changed in increments of $300 \mathrm{GeV}$. $K$-factor is set to be $K=1.6$

\subsection{Constraints}

When $m_{s}=300 \mathrm{GeV}$, the $95 \% \mathrm{CL}$ upper bound at $\sqrt{s}=$ $13 \mathrm{TeV}$ is $\sigma(s(\mathrm{ggF}) \rightarrow Z Z \rightarrow 4 l) 13 \mathrm{TeV} \lesssim 0.8 \mathrm{fb}[104]$; see also Fig. 6. The corresponding excluded region is plotted in Fig. 10.
Currently, the strongest direct constraint on the di-Higgs resonance at $m_{s}=300 \mathrm{GeV}$ comes from the $\sqrt{s}=8 \mathrm{TeV}$ data in the $b \bar{b} \gamma \gamma$ final state at CMS [112] and in $b \bar{b} \tau \tau$ at ATLAS [113]:

$\sigma(p p \rightarrow s \rightarrow h h)_{8 \mathrm{TeV}}< \begin{cases}1.1 \mathrm{pb} & (b \bar{b} \gamma \gamma \text { at CMS }), \\ 1.7 \mathrm{pb} & (b \bar{b} \tau \tau \text { at ATLAS }),\end{cases}$ 


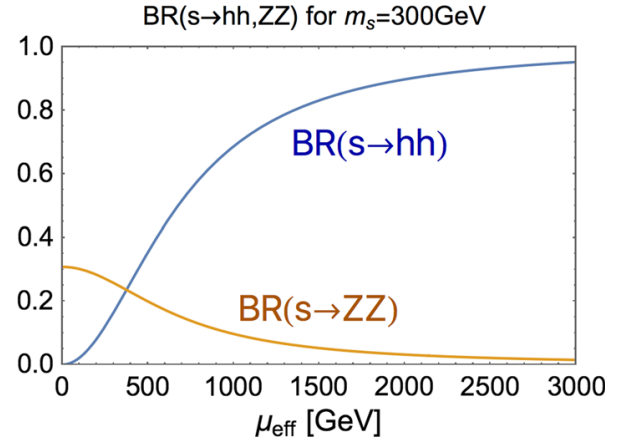

Fig. 9 Branching ratios $\mathrm{BR}(s \rightarrow h h)$ and $\mathrm{BR}(s \rightarrow Z Z)$ at $m_{s}=$ $300 \mathrm{GeV}$ as functions of $\mu_{\text {eff }}$

at the $95 \%$ CL. The preferred value (33) is still within this limit.

We note that the current limit for the $m_{s}=300 \mathrm{GeV}$ resonance search at $\sqrt{s}=13 \mathrm{TeV}$ is from the $b \bar{b} \gamma \gamma$ final state at ATLAS [113] and from $b \bar{b} b b$ at CMS [112]:

$\sigma(p p \rightarrow s \rightarrow h h)_{13 \mathrm{TeV}}< \begin{cases}5.5 \mathrm{pb} & (b \bar{b} \gamma \gamma \text { at ATLAS }), \\ 11 \mathrm{pb} & (b \bar{b} b b \text { at CMS }),\end{cases}$

at the $95 \% \mathrm{CL}$. This translates to the $\sqrt{s}=8 \mathrm{TeV}$ cross section:

$\sigma(p p \rightarrow s \rightarrow h h)_{8 \mathrm{TeV}}< \begin{cases}1.7 \mathrm{pb} & (b \bar{b} \gamma \gamma \text { at ATLAS }), \\ 3.5 \mathrm{pb} & (b \bar{b} b b \text { at CMS }) .\end{cases}$

This is weaker than the direct $8 \mathrm{TeV}$ bound (37).

The branching ratio for $s \rightarrow \gamma \gamma$ is ${ }^{9}$

$$
\begin{aligned}
& \mathrm{BR}(s \rightarrow \gamma \gamma) \sim 2.3 \times 10^{-3}\left[\frac{\alpha}{1 / 129}\right]^{2}\left[\frac{\mu_{\mathrm{eff}}}{800 \mathrm{GeV}}\right]^{-2} \\
& \quad \times\left[\frac{b_{\gamma}}{-8 / 9}\right]^{2}\left[\frac{m_{s}}{300 \mathrm{GeV}}\right]^{4}\left[\frac{\sin \theta}{0.01}\right]^{-2} .
\end{aligned}
$$

We see that the loop-suppressed decay into a di-photon is negligible compared to the tree-level decay via the interaction (4). For $m_{s}=300 \mathrm{GeV}$, the cross section at $\sqrt{s}=$ $13 \mathrm{TeV}$ is

$$
\begin{aligned}
& \sigma(p p \rightarrow s \rightarrow \gamma \gamma)_{13 \mathrm{TeV}} \sim 7.4 \mathrm{fb}\left[\frac{b_{g}}{-1 / 3}\right]^{2}\left[\frac{b_{\gamma}}{-8 / 9}\right]^{2} \\
& \times\left[\frac{\alpha_{s}}{0.1}\right]^{2}\left[\frac{\alpha}{1 / 129}\right]^{2}\left[\frac{\mu_{\mathrm{eff}}}{800 \mathrm{GeV}}\right]^{-2}\left[\frac{\sin \theta}{0.01}\right]^{-2} .
\end{aligned}
$$

We see that the loop-suppressed $\Gamma(s \rightarrow \gamma \gamma)$ becomes the same order as $\Gamma(s \rightarrow h h)$ when $\theta \lesssim 10^{-3}$ and that the region $\theta \lesssim 10^{-2}$ is excluded by the di-photon search, $\sigma(p p \rightarrow s \rightarrow$

\footnotetext{
9 The power of $m_{s}$ dependence is valid in the limit $m_{s} \gg 2 m_{h}$.
}

$\gamma \gamma)_{13 \mathrm{TeV}} \lesssim 10 \mathrm{fb}$ [105], for a typical set of parameters that explains the $300 \mathrm{GeV}$ excess; see also Sect. 4.3.

We comment on the case where the neutral scalar is charged under the $Z_{2}$ symmetry, $S \rightarrow-S$, or is extended to a complex scalar charged under an extra U(1), $S \rightarrow e^{i \varphi} S$. In such a model, the effective coupling in the small mixing limit becomes

$\mu_{\mathrm{eff}} \sim \frac{m_{s} f}{v} \lesssim \frac{m_{s}}{\eta}$

see Appendix B. That is, for a given $m_{s}$, there is an upper bound on the product $\mu_{\text {eff }} \eta: \mu_{\text {eff }} \eta \lesssim m_{s}$. On the other hand, the production cross section and the di-Higgs decay rate of $s$ are proportional to $\eta^{2}$ and $\mu_{\text {eff }}^{2}$, and hence there is a preferred value of $\mu_{\mathrm{eff}} \eta$ in order to account for the $2.4 \sigma$ excess by $m_{s}=300 \mathrm{GeV}$; see Fig. 10. In the $Z_{2}$ model and the $U(1)$ model, this preferred value exceeds the above upper bound. That is, they cannot account for the excess. A more rigorous proof can be found in Appendix B.

On the other hand, a singlet scalar that does not respect additional symmetry does not obey Eq. (42). For this reason, a singlet scalar without $Z_{2}$ symmetry is advantageous to enhance the di-Higgs signal in general and can explain the excess by $m_{s}=300 \mathrm{GeV}$.

\section{Summary and discussion}

We have studied a class of models in which the di-Higgs production is enhanced by the $s$-channel resonance of the neutral scalar that couples to a pair of gluons by the loop of heavy colored fermion or scalar. As such a colored particle, we have considered two types of possibilities:

- the vector-like fermionic partner of top or bottom quark, with which the neutral scalar may be identified as the dilaton in the quasi-conformal sector,

- the colored scalar which is either triplet (leptoquark), sextet (diquark), or octet (coloron).

We have presented the future prospect for the enhanced di-Higgs production in the LHC and beyond. Typically, the top/bottom partner models give a cross section $\sigma(p p \rightarrow s) \gtrsim$ $1 \mathrm{fb}$, which could be accessed by a luminosity of $\mathcal{O}\left(\mathrm{ab}^{-1}\right)$, for the scalar mass $m_{s} \lesssim 1.3 \mathrm{TeV}, 2 \mathrm{TeV}$, and $4 \mathrm{TeV}$ at the LHC, HE-LHC, and FCC, respectively.

We have examined the constraints from the direct searches for the di-Higgs signal and for a heavy colored particle, as well as the Higgs signal strengths in various production and decay channels. Typically small and large mixing regions are excluded by the di-photon resonance search and by the Higgs signal-strength bounds, respectively. The region of small $\mu_{\text {eff }}$ is excluded by the di-photon search as well as by the $s \rightarrow$ $Z Z \rightarrow 4 l$ channel. 

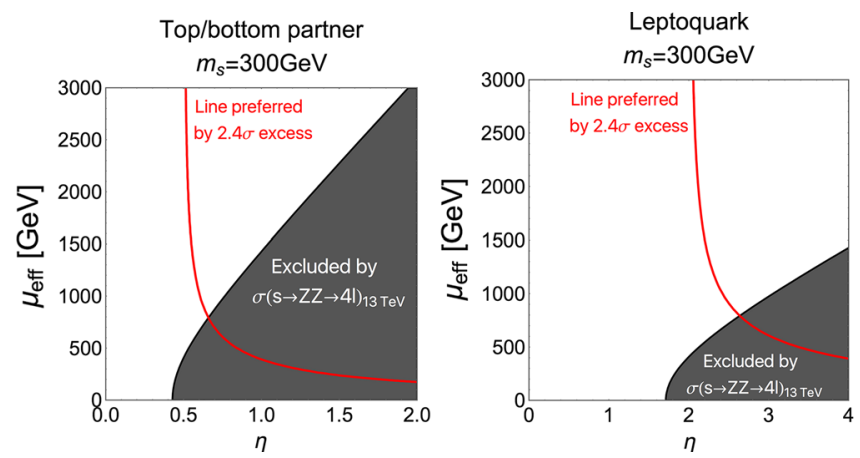

Fig. 10 In each panel, the line corresponds to the preferred contour to explain the $2.4 \sigma$ excess at $m_{s}=300 \mathrm{GeV}$, and the shaded region is excluded at the $95 \% \mathrm{CL}$ by $\sigma(Z Z \rightarrow 4 l)_{13 \mathrm{TeV}}$. The $K$-factor is set

We also show a possible explanation of the $2.4 \sigma$ excess of the di-Higgs signal in the $b \bar{b} \gamma \gamma$ final state, reported by the ATLAS experiment. We have shown that the $Z_{2}$ model explained in Appendix B cannot account for the excess, while the general model in Appendix A can. A typical benchmark point which evades all the bounds and can explain the excess is

$\mu_{\mathrm{eff}} \sim 1 \mathrm{TeV}, \quad \eta \sim\left\{\begin{array}{ll}0.6 & \text { top/bottom partner, } \\ 2.4 & \text { leptoquark, } \\ 0.5 & \text { diquark, } \\ 0.4 & \text { coloron, }\end{array} \quad \sin \theta \sim 0.1\right.$.

For the top/bottom partner $T, B$, the required value to explain the $2.4 \sigma$ excess for the Yukawa coupling is rather large $y_{F} N_{F} \gtrsim 2.2$, where $N_{F}$ is the number of $F=T, B$ introduced. For the colored scalar $\phi$, required value of the neutral scalar VEV, $f=\langle S\rangle$, are

$f \kappa_{\phi} N_{\phi} \gtrsim \begin{cases}5-13 \mathrm{TeV} & \begin{array}{l}\text { leptoquark, depending on } \\ \end{array} \\ \text { possible decay channels, } \\ 106 \mathrm{TeV} & \text { diquark, } \\ 54 \mathrm{TeV} & \text { coloron, }\end{cases}$

where $\kappa_{\phi}$ and $N_{\phi}$ are the quartic coupling between the colored and neutral scalars and the number of colored scalar introduced, respectively.

In this paper, we have restricted ourselves to the case where the colored particle running in the blob in Fig. 1 are $S U(2)_{L}$ singlet. Cases for doublet, triplet, etc., which could be richer in phenomenology, will be presented elsewhere. We have assumed $M_{F}, M_{\phi} \gtrsim m_{s}$ to justify integrating out the colored particle. It would be worth including loop functions to extend the region of study toward $M_{F}, M_{\phi} \lesssim m_{s}$. A full collider simulation of this model for HL-LHC and FCC would be worth studying. A theoretical background of this type of the neutral scalar assisted by the colored
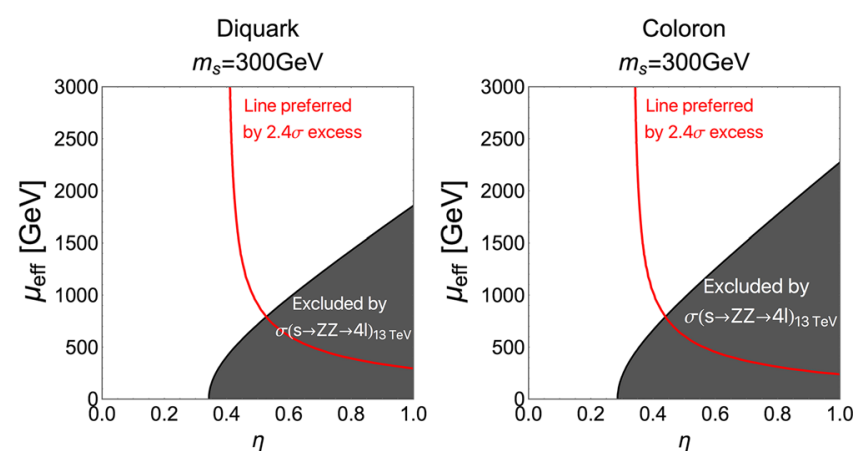

to be $K=1.6$. The region $10^{-4} \lesssim \theta^{2} \ll 1$ is assumed. Note that the plotted region of $\eta$ in horizontal axis differs panel by panel

fermion/scalar is worth pushing, such as the dilaton model and the leptoquark model with spontaneous $B-L$ symmetry breaking.

Acknowledgements The work of K. Nakamura and K.O. are partially supported by the JSPS KAKENHI Grant No. 26800156 and Nos. 15K05053, 23104009, respectively. S.C.P. and Y.Y. are supported by the National Research Foundation of Korea (NRF) grant funded by the Korean government (MSIP) (No. 2016R1A2B2016112). K. Nishiwaki would like to thank David London and the Group of Particle Physics of Université de Montréal for the kind hospitality at the final stage of this work.

Open Access This article is distributed under the terms of the Creative Commons Attribution 4.0 International License (http://creativecomm ons.org/licenses/by/4.0/), which permits unrestricted use, distribution, and reproduction in any medium, provided you give appropriate credit to the original author(s) and the source, provide a link to the Creative Commons license, and indicate if changes were made.

Funded by $\mathrm{SCOAP}^{3}$.

\section{Appendix A: General scalar potential}

We write down the most general renormalizable potential including the SM Higgs $H$ and the singlet $S$ :

$V=V_{S}+V_{H}+V_{S H}$,

with $^{10}$

$$
\begin{aligned}
& V_{S}=\frac{m_{S}^{2}}{2} S^{2}+\frac{\mu_{S}}{3 !} S^{3}+\frac{\lambda_{S}}{4 !} S^{4}, \\
& V_{H}=m_{H}^{2}|H|^{2}+\frac{\lambda_{H}}{2}|H|^{4}, \\
& V_{S H}=\mu S|H|^{2}+\frac{\kappa}{2} S^{2}|H|^{2},
\end{aligned}
$$

where $m_{S}^{2}$ and $m_{H}^{2}$ are (potentially negative) mass-squared parameters; $\lambda_{S}, \kappa, \lambda_{H}$ are dimensionless constants; $\mu_{S}$ and

10 Our $\lambda_{H}$ differs from the conventionally used $\lambda$ by $\lambda_{H}=2 \lambda$, with $\lambda=m_{h}^{2} / 2 v^{2} \simeq 0.13$ in the SM; see e.g. Refs. [114,115]. 
$\mu$ are real parameters of the mass dimension unity; and the tadpole term of $S$ is removed by the field redefinition $S \rightarrow$ $S+$ const. The $Z_{2}$ model corresponds to setting $\mu_{S}=\mu=0$, which is prohibited by the $Z_{2}$ symmetry: $S \rightarrow-S$.

The vacuum condition reads

$$
\begin{aligned}
& \lambda_{H}|H|^{2}+\mu S+\frac{\kappa}{2} S^{2}=-m_{H}^{2}, \\
& |H|^{2}(\mu+\kappa S)+\frac{\mu_{S}}{2} S^{2}+\frac{\lambda_{S}}{3 !} S^{3}=-m_{S}^{2} S .
\end{aligned}
$$

Using this vacuum condition, and putting Eqs. (1) and (2), we can always rewrite $m_{H}^{2}$ and $m_{S}^{2}$ in terms of $v, f$, and other parameters. The mixing angle can be written as

$$
\tan 2 \theta=\frac{v(f \kappa+\mu)}{\frac{\lambda_{S}}{3 !} f^{2}-\frac{\lambda_{H}}{2} v^{2}+\frac{\mu_{S}}{4} f-\frac{\mu}{4} \frac{v^{2}}{f}}
$$

Now the effective coupling in Eq. (4) is written as

$$
\begin{aligned}
\mu_{\mathrm{eff}}= & (\kappa f+\mu) \frac{\cos ^{3} \theta}{\sin \theta}+v\left(3 \lambda_{H}-2 \kappa\right) \cos ^{2} \theta \\
& +\left[f\left(\lambda_{S}-2 \kappa\right)-2 \mu+\mu_{S}\right] \cos \theta \sin \theta+\kappa v \sin ^{2} \theta .
\end{aligned}
$$

In the small mixing limit $\theta^{2} \ll 1$, we obtain

$\mu_{\mathrm{eff}}=\frac{\kappa f+\mu}{\theta}+v\left(3 \lambda_{H}-2 \kappa\right)+\mathcal{O}(\theta)$.

We note that the first term also goes to a constant for fixed $v, f$ because of Eq. (51): $(\kappa f+\mu) \propto \theta$. More explicitly,

$\mu_{\mathrm{eff}} \rightarrow \frac{\lambda_{S}}{3} \frac{f^{2}}{v}+2\left(\lambda_{H}-\kappa\right) v+\frac{\mu_{S}}{2} \frac{f}{v}-\frac{\mu}{2} \frac{v}{f}$

as $\theta \rightarrow 0$. That is, the $s h h$ coupling vanishes in the small mixing limit: $\mu_{\text {eff }} \sin \theta \rightarrow 0$. Let us emphasize that this is a general feature since the $s h h$ coupling necessarily requires the non-zero mixing term $v s h$ that is obtained by the replacement $h \rightarrow v$. In order to take this feature into account, we have parametrized the effective coupling as in Eq. (4).

The mass eigenvalues satisfy the relations

$$
\begin{aligned}
m_{s}^{2}+m_{h}^{2} & =\lambda_{H} v^{2}+\frac{\lambda_{S}}{3} f^{2}-\frac{\mu}{2} \frac{v^{2}}{f}+\frac{\mu_{S}}{2} f, \\
m_{s}^{2} m_{h}^{2}= & (f v)^{2}\left[\frac{\lambda_{S} \lambda_{H}}{3}-\kappa^{2}\right. \\
& \left.-\frac{\mu}{f}\left(2 \kappa-\frac{\lambda_{H}}{2} \frac{\mu_{S}}{\mu}+\frac{\mu}{f}+\frac{\lambda_{H}}{2} \frac{v^{2}}{f^{2}}\right)\right],
\end{aligned}
$$

where we suppose $m_{s}>m_{h} \simeq 125 \mathrm{GeV}$. The tachyon free condition is that the right hand sides of Eqs. (55) and (56) are positive. Also, from the condition that the quartic terms are positive in the large field limit for any linear com- bination of two fields, we obtain $\lambda_{S}>0, \lambda_{H}>0$, and $\left(\frac{\lambda_{H} \lambda_{S}}{3}-\kappa^{2}\right)\left(\frac{\lambda_{H}}{2}+\frac{\lambda_{S}}{3 !}-\kappa\right)>0 . .^{11}$

In the model without the $Z_{2}$ symmetry, we can remove the parameters $\mu$ and $\mu_{S}$ using Eqs. (55) and (56). Then the mixing angle (51) may be rewritten as

$\tan 2 \theta=\frac{\sqrt{\lambda_{H} v^{2}-m_{h}^{2}} \sqrt{m_{s}^{2}-\lambda_{H} v^{2}}}{\frac{m_{s}^{2}+m_{h}^{2}}{2}-\lambda_{H} v^{2}}$.

Such a solution for $\lambda_{H}>0$ exists only when

$\frac{m_{h}^{2}}{v^{2}}<\lambda_{H}<\frac{m_{s}^{2}}{v^{2}}$

We see that the small mixing limit corresponds to $\lambda_{H} \searrow$ $m_{h}^{2} / v^{2}$. Also one may remove $\mu, \mu_{S}$ from the small mixing limit (54):

$$
\begin{aligned}
& \mu_{\mathrm{eff}} \rightarrow v\left(\left(\lambda_{H}-2 \kappa\right)+\frac{m_{s}^{2}+m_{h}^{2}}{v^{2}}\right) \\
& =v\left(-2 \kappa+\frac{m_{s}^{2}+2 m_{h}^{2}}{v^{2}}\right),
\end{aligned}
$$

where we used Eqs. (55) and (56) in the first step, and substituted the $\lambda_{H} \searrow m_{h}^{2} / v^{2}$ limit in the next step. We see that the Higgs-singlet mixing $\kappa$ remains a free parameters even in the small mixing limit.

If we want to explain the $b \bar{b} \gamma \gamma$ excess [15], we set $m_{s} \simeq$ $300 \mathrm{GeV}$ and get

$\frac{m_{s}^{2}+m_{h}^{2}}{2} \simeq(230 \mathrm{GeV})^{2}, \quad m_{s}^{2} m_{h}^{2} \simeq(190 \mathrm{GeV})^{4}$

Even in the small mixing limit, $\mu_{\text {eff }}$ in Eq. (59) with $m_{s}=$ $300 \mathrm{GeV}$ can be as large as $\mu_{\mathrm{eff}} \simeq 1 \mathrm{TeV}(2 \mathrm{TeV})$ for $\kappa=-1$ $(-3)$, which is well within the current experimental bound; see Fig. 10; if we are happy with an extremely large value, say $\kappa=-4 \pi$, we may push it up to $\mu_{\text {eff }} \simeq 6.7 \mathrm{TeV}$.

\section{Appendix B: $Z_{2}$ model}

We consider the $Z_{2}$ model with $\mu=\mu_{S}=0$. The discussion is parallel to Appendix A. The mixing angle reads

$\tan 2 \theta=\frac{\kappa v}{\frac{\lambda_{S}}{3 !} f-\frac{\lambda_{H}}{2} \frac{v^{2}}{f}}$.

\footnotetext{
11 When we allow higher dimensional operators such as $S^{6}$, this vacuum stability condition can be violated. In this analysis, we restrict ourselves to the potential up to quartic order terms, and we assume that this condition is met.
} 
Especially in the limit $v \ll f$, we get $\tan 2 \theta \rightarrow \frac{6 \kappa}{\lambda_{S}} \frac{v}{f}$. Equations (55) and (56) may be solved e.g. as

$$
\begin{gathered}
f^{2}=\frac{\left(\lambda_{H} v^{2}-m_{h}^{2}\right)\left(m_{s}^{2}-\lambda_{H} v^{2}\right)}{\kappa^{2} v^{2}}, \\
\lambda_{S}=\frac{3 \kappa^{2} v^{2}\left(m_{h}^{2}+m_{s}^{2}-\lambda_{H} v^{2}\right)}{\left(\lambda_{H} v^{2}-m_{h}^{2}\right)\left(m_{s}^{2}-\lambda_{H} v^{2}\right)} .
\end{gathered}
$$

For $\lambda_{H}>0$, the solution with $m_{s}>m_{h}>0$ again exists when and only when the condition (58) is met. This condition also ensures $\lambda_{S}$ to be positive. Putting Eq. (62) into Eq. (61), we again obtain Eq. (57).

Finally, the small mixing limit of the effective coupling becomes

$\mu_{\mathrm{eff}} \rightarrow v\left(\lambda_{H}+\frac{m_{s}^{2}+m_{h}^{2}}{v^{2}}\right)=\frac{m_{s}^{2}+2 m_{h}^{2}}{v}$.

If we want to set $m_{s}=300 \mathrm{GeV}$, we get $\mu_{\mathrm{eff}} \simeq 490 \mathrm{GeV}$ in the small mixing limit $\theta^{2} \ll 1$, which is already excluded by the $s \rightarrow Z Z \rightarrow 4 l$ search; see Fig. 10 . The $Z_{2}$ model cannot explain the $2.4 \sigma$ excess reported by ATLAS. For larger values of $m_{s}$, the $Z_{2}$ model is still viable.

\section{Appendix C: Yukawa interaction between colored scalar and SM particles}

For the scalar in the fundamental representation $\phi_{\mathbf{3}}$, the possible Yukawa interactions are

$$
\left(\phi_{\mathbf{3}}\right)^{*} \overline{\left(q_{\mathrm{L}}\right)^{c}}{ }_{i} \cdot l_{\mathrm{L}}^{i}, \quad\left(\phi_{\mathbf{3}}\right)^{*} \overline{\left(u_{\mathrm{R}}\right)^{c}} e_{\mathrm{R}}, \quad\left(\phi_{\mathbf{3}}\right)^{*} \overline{\left(d_{\mathrm{R}}\right)^{c}} e_{\mathrm{R}},
$$

depending on the hypercharge of $\phi_{3}:-1 / 3,-1 / 3$, and $-4 / 3$, respectively. The superscript ${ }^{c}$ denotes the charge conjugation.

We note that we can in principle write down the following diquark interactions:

$$
\begin{gathered}
\epsilon^{a b c} \epsilon^{i j}\left(\phi_{\mathbf{3}}\right)_{a} \overline{\left(q_{\mathrm{L}}\right)^{c}}{ }_{b i}\left(q_{\mathrm{L}}\right)_{c j}, \quad \epsilon^{a b c}\left(\phi_{\mathbf{3}}\right)_{a} \overline{\left(u_{\mathrm{R}}\right)^{c}}{ }_{b}\left(u_{\mathrm{R}}\right)_{c}, \\
\epsilon^{a b c}\left(\phi_{\mathbf{3}}\right)_{a} \overline{\left(d_{\mathrm{R}}\right)^{c}}{ }_{b}\left(d_{\mathrm{R}}\right)_{c}, \quad \epsilon^{a b c}\left(\phi_{\mathbf{3}}\right)_{a} \overline{\left(u_{\mathrm{R}}\right)^{c}}{ }_{b}\left(d_{\mathrm{R}}\right)_{c},
\end{gathered}
$$

depending on the hypercharge of $\phi_{3}:-1 / 3,-4 / 3,2 / 3$ and $-1 / 3$, respectively, where $a, b, c$ and $i, j$ represent the indices of the $S U(3)_{C}$ and $S U(2)_{L}$ fundamental representations, respectively, and $\epsilon$ is the totally antisymmetric tensor. The coexistence of the leptoquark and the diquark interactions leads to rapid proton decay. Since the diquark interactions are strongly restricted compared with the leptoquark in direct searches in hadron colliders, we focus on the situation that only the leptoquark interactions are switched on. The diquark interactions can be forbidden e.g. by the $B-L$ symmetry.
For the symmetric scalar $\phi_{\mathbf{6}}$, a possible Yukawa is either one of

$$
\begin{gathered}
\overline{\left(u_{\mathrm{R}}\right)_{a}^{c}}\left(\phi_{\mathbf{6}}\right)^{* a b}\left(u_{\mathrm{R}}\right)_{b}, \quad \overline{\left(d_{\mathrm{R}}\right)_{a}^{c}}\left(\phi_{\mathbf{6}}\right)^{* a b}\left(u_{\mathrm{R}}\right)_{b}, \\
\overline{\left(d_{\mathrm{R}}\right)_{a}^{c}}\left(\phi_{\mathbf{6}}\right)^{* a b}\left(d_{\mathrm{R}}\right)_{b}, \quad \epsilon^{i j} \overline{\left(q_{\mathrm{L}}\right)_{a i}^{c}}\left(\phi_{\mathbf{6}}\right)^{* a b}\left(q_{\mathrm{L}}\right)_{b j},
\end{gathered}
$$

depending on the hypercharge of $\phi_{6}: 4 / 3,1 / 3,-2 / 3$, and $1 / 3$, respectively.

For adjoint scalar, a possible lowest-dimensional Yukawa is either one of

$$
\begin{gathered}
\frac{1}{\Lambda}{\overline{u_{\mathrm{R}}}}^{a}\left(\phi_{\mathbf{8}}\right)_{a}{ }^{b}\left(q_{\mathrm{L}}\right)_{b i} \epsilon^{i j} H_{j}, \quad \frac{1}{\Lambda}{\overline{u_{\mathrm{R}}}}^{a}\left(\phi_{\mathbf{8}}\right)_{a}{ }^{b}\left(q_{\mathrm{L}}\right)_{b i}\left(H^{*}\right)^{i}, \\
\frac{1}{\Lambda}{\overline{\bar{R}_{\mathrm{R}}}}^{a}\left(\phi_{\mathbf{8}}\right)_{a}{ }^{b}\left(q_{\mathrm{L}}\right)_{b i} \epsilon^{i j} H_{j}, \quad \frac{1}{\Lambda}{\overline{d_{\mathrm{R}}}}^{a}\left(\phi_{\mathbf{8}}\right)_{a}{ }^{b}\left(q_{\mathrm{L}}\right)_{b i}\left(H^{*}\right)^{i},
\end{gathered}
$$

depending on the hypercharge of $\phi_{\mathbf{8}}: 0,-1,-1$, and 0 , respectively, where we have assigned $Y_{H}=+1 / 2$ and $\Lambda$ denotes an ultraviolet cutoff scale.

\section{References}

1. E.W.N. Glover, J.J. van der Bij, Higgs boson pair production via gluon fusion. Nucl. Phys. B 309, 282-294 (1988)

2. O.J.P. Eboli, G.C. Marques, S.F. Novaes, A.A. Natale, Twin Higgs boson production. Phys. Lett. B 197, 269-272 (1987)

3. S. Dawson, S. Dittmaier, M. Spira, Neutral Higgs boson pair production at hadron colliders: QCD corrections. Phys. Rev. D 58, 115012 (1998). arXiv:hep-ph/9805244

4. U. Baur, T. Plehn, D.L. Rainwater, Determining the Higgs boson selfcoupling at hadron colliders. Phys. Rev. D 67, 033003 (2003). arXiv:hep-ph/0211224

5. U. Baur, T. Plehn, D.L. Rainwater, Probing the Higgs selfcoupling at hadron colliders using rare decays. Phys. Rev. D 69, 053004 (2004). arXiv:hep-ph/0310056

6. D.Y. Shao, C.S. Li, H.T. Li, J. Wang, Threshold resummation effects in Higgs boson pair production at the LHC. JHEP 07, 169 (2013). arXiv:1301.1245

7. J. Grigo, J. Hoff, K. Melnikov, M. Steinhauser, On the Higgs boson pair production at the LHC. Nucl. Phys. B 875, 1-17 (2013). arXiv: 1305.7340

8. D. de Florian, J. Mazzitelli, Higgs boson pair production at nextto-next-to-leading order in QCD. Phys. Rev. Lett. 111, 201801 (2013). arXiv:1309.6594

9. G. Degrassi, P.P. Giardino, R. Groeber, On the two-loop virtual QCD corrections to Higgs boson pair production in the Standard Model. Eur. Phys. J. C 76(7), 411 (2016). arXiv:1603.00385

10. S. Borowka, N. Greiner, G. Heinrich, S.P. Jones, M. Kerner, J. Schlenk, T. Zirke, Full top quark mass dependence in Higgs boson pair production at NLO. JHEP 10, 107 (2016). arXiv: 1608.04798

11. G. Ferrera, J. Pires, Transverse-momentum resummation for Higgs boson pair production at the LHC with top-quark mass effects. JHEP 139, 1702 (2017). arXiv:1609.01691

12. J. Baglio, A. Djouadi, R. Grober, M.M. Muhlleitner, J. Quevillon, M. Spira, The measurement of the Higgs self-coupling at the LHC: theoretical status. JHEP 04, 151 (2013). arXiv:1212.5581

13. N. Craig, J. Galloway, S. Thomas, Searching for signs of the second Higgs doublet (2013). arXiv:1305.2424

14. J. Baglio, O. Eberhardt, U. Nierste, M. Wiebusch, Benchmarks for Higgs pair production and heavy Higgs boson searches in the 
two-Higgs-doublet model of type II. Phys. Rev. D 90(1), 015008 (2014). arXiv:1403.1264

15. Atlas, G. Aad et al., Search for Higgs boson pair production in the $\gamma \gamma b \bar{b}$ final state using $p p$ collision data at $\sqrt{s}=8 \mathrm{TeV}$ from the ATLAS detector. Phys. Rev. Lett. 114(8), 081802 (2015). arXiv: 1406.5053

16. B. Hespel, D. Lopez-Val, E. Vryonidou, Higgs pair production via gluon fusion in the two-Higgs-doublet model. JHEP 09, 124 (2014). arXiv:1407.0281

17. V. Barger, L.L. Everett, C.B. Jackson, A.D. Peterson, G. Shaughnessy, Measuring the two-Higgs doublet model scalar potential at LHC14. Phys. Rev. D 90(9), 095006 (2014). arXiv:1408.2525

18. L.-C. Lu, C. Du, Y. Fang, H.-J. He, H. Zhang, Searching heavier Higgs boson via di-Higgs production at LHC run-2. Phys. Lett. B 755, 509-522 (2016). arXiv: 1507.02644

19. G.C. Dorsch, S.J. Huber, K. Mimasu, J.M. No, Hierarchical versus degenerate 2HDM: the LHC run 1 legacy at the onset of run 2. Phys. Rev. D 93(11), 115033 (2016). arXiv:1601.04545

20. F. Kling, J.M. No, S. Su, Anatomy of exotic Higgs decays in 2HDM. JHEP 09, 093 (2016). arXiv: 1604.01406

21. L. Bian, N. Chen, Higgs pair productions in the CP-violating twoHiggs-doublet model. JHEP 09, 069 (2016). arXiv:1607.02703

22. Z.-L. Han, R. Ding, Y. Liao, LHC phenomenology of the type II seesaw mechanism: observability of neutral scalars in the nondegenerate case. Phys. Rev. D 92(3), 033014 (2015). arXiv: 1506.08996

23. G.D. Kribs, A. Martin, Enhanced di-Higgs production through light colored scalars. Phys. Rev. D 86, 095023 (2012). arXiv: 1207.4496

24. S. Dawson, E. Furlan, I. Lewis, Unravelling an extended quark sector through multiple Higgs production? Phys. Rev. D 87(1), 014007 (2013). arXiv: 1210.6663

25. A. Pierce, J. Thaler, L.-T. Wang, Disentangling dimension six operators through di-Higgs boson production. JHEP 05, 070 (2007). arXiv:hep-ph/0609049

26. S. Kanemura, K. Tsumura, Effects of the anomalous Higgs couplings on the Higgs boson production at the Large Hadron Collider. Eur. Phys. J. C 63, 11-21 (2009). arXiv:0810.0433

27. M.J. Dolan, C. Englert, M. Spannowsky, Higgs self-coupling measurements at the LHC. JHEP 10, 112 (2012). arXiv:1206.5001

28. K. Nishiwaki, S. Niyogi, A. Shivaji, $t t H$ anomalous coupling in double Higgs production. JHEP 04, 011 (2014). arXiv:1309.6907

29. C.-R. Chen, I. Low, Double take on new physics in double Higgs boson production. Phys. Rev. D 90(1), 013018 (2014). arXiv: 1405.7040

30. N. Liu, S. Hu, B. Yang, J. Han, Impact of top-Higgs couplings on di-Higgs production at future colliders. JHEP 01, 008 (2015). arXiv: 1408.4191

31. M. Slawinska, W. van den Wollenberg, B. van Eijk, S. Bentvelsen, Phenomenology of the trilinear Higgs coupling at proton-proton colliders (2014). arXiv: 1408.5010

32. F. Goertz, A. Papaefstathiou, L.L. Yang, J. Zurita, Higgs boson pair production in the $\mathrm{D}=6$ extension of the SM. JHEP 04, 167 (2015). arXiv: 1410.3471

33. A. Azatov, R. Contino, G. Panico, M. Son, Effective field theory analysis of double Higgs boson production via gluon fusion. Phys. Rev. D 92(3), 035001 (2015). arXiv:1502.00539

34. C.-T. Lu, J. Chang, K. Cheung, J.S. Lee, An exploratory study of Higgs-boson pair production. JHEP 08, 133 (2015). arXiv: 1505.00957

35. A. Carvalho, M. Dall'Osso, T. Dorigo, F. Goertz, C.A. Gottardo, M. Tosi, Higgs pair production: choosing benchmarks with cluster analysis. JHEP 04, 126 (2016). arXiv: 1507.02245

36. D. de Florian, M. Grazzini, C. Hanga, S. Kallweit, J.M. Lindert, P. Maierhoefer, J. Mazzitelli, D. Rathlev, Differential Higgs boson pair production at next-to-next-to-leading order in QCD. JHEP 09, 151 (2016). arXiv:1606.09519

37. M. Gorbahn, U. Haisch, Indirect probes of the trilinear Higgs coupling: $g g \rightarrow h$ and $h \rightarrow \gamma \gamma$. JHEP 10, 094 (2016). arXiv: 1607.03773

38. A. Carvalho, M. Dall'Osso, P. De Castro Manzano, T. Dorigo, F. Goertz, M. Gouzevich, M. Tosi, Analytical parametrization and shape classification of anomalous $\mathrm{HH}$ production in the EFT approach (2016). arXiv:1608.06578

39. Q.-H. Cao, G. Li, B. Yan, D.-M. Zhang, H. Zhang, Double Higgs production at the $14 \mathrm{TeV}$ LHC and the $100 \mathrm{TeV}$ pp-collider (2016). arXiv: 1611.09336

40. M.J. Dolan, C. Englert, M. Spannowsky, New physics in LHC Higgs boson pair production. Phys. Rev. D 87(5), 055002 (2013). arXiv: 1210.8166

41. R. Contino, C. Grojean, M. Moretti, F. Piccinini, R. Rattazzi, Strong double Higgs production at the LHC. JHEP 05, 089 (2010). arXiv: 1002.1011

42. R. Grober, M. Muhlleitner, Composite Higgs boson pair production at the LHC. JHEP 06, 020 (2011). arXiv:1012.1562

43. R. Contino, M. Ghezzi, M. Moretti, G. Panico, F. Piccinini, A. Wulzer, Anomalous couplings in double Higgs production. JHEP 08, 154 (2012). arXiv:1205.5444

44. R. Grober, M. Muhlleitner, M. Spira, Signs of composite Higgs pair production at next-to-leading order. JHEP 06, 080 (2016). arXiv: 1602.05851

45. J.-J. Liu, W.-G. Ma, G. Li, R.-Y. Zhang, H.-S. Hou, Higgs boson pair production in the little Higgs model at hadron collider. Phys. Rev. D 70, 015001 (2004). arXiv:hep-ph/0404171

46. C.O. Dib, R. Rosenfeld, A. Zerwekh, Double Higgs production and quadratic divergence cancellation in little Higgs models with T parity. JHEP 05, 074 (2006). arXiv:hep-ph/0509179

47. L. Wang, W. Wang, J.M. Yang, H. Zhang, Higgs-pair production in littlest Higgs model with T-parity. Phys. Rev. D 76, 017702 (2007). arXiv:0705.3392

48. N. Craig, A. Katz, M. Strassler, R. Sundrum, Naturalness in the dark at the LHC. JHEP 07, 105 (2015). arXiv:1501.05310

49. C.-Y. Chen, S. Dawson, I.M. Lewis, Exploring resonant di-Higgs boson production in the Higgs singlet model. Phys. Rev. D 91(3), 035015 (2015). arXiv: 1410.5488

50. T. Robens, T. Stefaniak, Status of the Higgs singlet extension of the standard model after LHC run 1. Eur. Phys. J. C 75, 104 (2015). arXiv: 1501.02234

51. V. Martin Lozano, J.M. Moreno, C.B. Park, Resonant Higgs boson pair production in the $h h \rightarrow b \bar{b} W W \rightarrow b \bar{b} \ell^{+} v \ell^{-} \bar{v}$ decay channel. JHEP 08, 004 (2015). arXiv: 1501.03799

52. A. Falkowski, C. Gross, O. Lebedev, A second Higgs from the Higgs portal. JHEP 05, 057 (2015). arXiv:1502.01361

53. D. Buttazzo, F. Sala, A. Tesi, Singlet-like Higgs bosons at present and future colliders. JHEP 11, 158 (2015). arXiv: 1505.05488

54. S. Dawson, I.M. Lewis, NLO corrections to double Higgs boson production in the Higgs singlet model. Phys. Rev. D 92(9), 094023 (2015). arXiv: 1508.05397

55. T. Robens, T. Stefaniak, LHC benchmark scenarios for the real Higgs singlet extension of the standard model. Eur. Phys. J. C 76(5), 268 (2016). arXiv:1601.07880

56. G. Dupuis, Collider constraints and prospects of a scalar singlet extension to Higgs portal dark matter. JHEP 07, 008 (2016). arXiv: 1604.04552

57. S. Banerjee, B. Batell, M. Spannowsky, Invisible decays in Higgs pair production. Phys. Rev. D95(3), 035009 (2017). arXiv: 1608.08601

58. T. Plehn, M. Spira, P.M. Zerwas, Pair production of neutral Higgs particles in gluon-gluon collisions. Nucl. Phys. B 479, 46-64 (1996). arXiv:hep-ph/9603205 [Erratum: Nucl. Phys. B 531, 655 (1998)] 
59. A. Djouadi, W. Kilian, M. Muhlleitner, P.M. Zerwas, Production of neutral Higgs boson pairs at LHC. Eur. Phys. J. C 10, 45-49 (1999). arXiv:hep-ph/9904287

60. J. Cao, Z. Heng, L. Shang, P. Wan, J.M. Yang, Pair production of a $125 \mathrm{GeV}$ Higgs boson in MSSM and NMSSM at the LHC. JHEP 04, 134 (2013). arXiv:1301.6437

61. C. Han, X. Ji, L. Wu, P. Wu, J.M. Yang, Higgs pair production with SUSY QCD correction: revisited under current experimental constraints. JHEP 04, 003 (2014). arXiv: 1307.3790

62. B. Bhattacherjee, A. Choudhury, Role of supersymmetric heavy Higgs boson production in the self-coupling measurement of 125 GeV Higgs boson at the LHC. Phys. Rev. D 91, 073015 (2015). arXiv: 1407.6866

63. J. Cao, D. Li, L. Shang, P. Wu, Y. Zhang, Exploring the Higgs sector of a most natural NMSSM and its prediction on Higgs pair production at the LHC. JHEP 12, 026 (2014). arXiv: 1409.8431

64. A. Djouadi, L. Maiani, A. Polosa, J. Quevillon, V. Riquer, Fully covering the MSSM Higgs sector at the LHC. JHEP 06, 168 (2015). arXiv: 1502.05653

65. B. Batell, S. Jung, Probing light stops with stoponium. JHEP 07, 061 (2015). arXiv: 1504.01740

66. L. Wu, J.M. Yang, C.-P. Yuan, M. Zhang, Higgs self-coupling in the MSSM and NMSSM after the LHC run 1. Phys. Lett. B 747, 378-389 (2015). arXiv:1504.06932

67. B. Batell, M. McCullough, D. Stolarski, C.B. Verhaaren, Putting a stop to di-Higgs modifications. JHEP 09, 216 (2015). arXiv: 1508.01208

68. R. Costa, M. Muehlleitner, M.O.P. Sampaio, R. Santos, Singlet extensions of the standard model at LHC run 2: benchmarks and comparison with the NMSSM. JHEP 06, 034 (2016). arXiv: 1512.05355

69. A. Agostini, G. Degrassi, R. Grueber, P. Slavich, NLO-QCD corrections to Higgs pair production in the MSSM. JHEP 04, 106 (2016). arXiv: 1601.03671

70. A. Hammad, S. Khalil, S. Moretti, LHC signals of a B-L supersymmetric standard model CP-even Higgs boson. Phys. Rev. D 93(11), 115035 (2016). arXiv:1601.07934

71. S. Biswas, E.J. Chun, P. Sharma, Di-Higgs signatures from Rparity violating supersymmetry as the origin of neutrino mass. JHEP 12, 062 (2016). arXiv:1604.02821

72. CMS, V. Khachatryan et al., Search for two Higgs bosons in final states containing two photons and two bottom quarks in protonproton collisions at $8 \mathrm{TeV}$. Phys. Rev. D 94(5), 052012 (2016). arXiv:1603.06896

73. E. Asakawa, D. Harada, S. Kanemura, Y. Okada, K. Tsumura, Higgs boson pair production in new physics models at hadron, lepton, and photon colliders. Phys. Rev. D 82, 115002 (2010). arXiv: 1009.4670

74. A. Papaefstathiou, L.L. Yang, J. Zurita, Higgs boson pair production at the LHC in the $b \bar{b} W^{+} W^{-}$channel. Phys. Rev. D 87(1), 011301 (2013). arXiv: 1209.1489

75. M. Klute, R. Lafaye, T. Plehn, M. Rauch, D. Zerwas, Measuring Higgs couplings at a linear collider. Europhys. Lett. 101, 51001 (2013). arXiv:1301.1322

76. F. Goertz, A. Papaefstathiou, L.L. Yang, J. Zurita, Higgs boson self-coupling measurements using ratios of cross sections. JHEP 06, 016 (2013). arXiv:1301.3492

77. A.J. Barr, M.J. Dolan, C. Englert, M. Spannowsky, Di-Higgs final states augMT2ed - selecting $h h$ events at the high luminosity LHC. Phys. Lett. B 728, 308-313 (2014). arXiv:1309.6318

78. N. Chen, C. Du, Y. Fang, L.-C. Lü, LHC searches for the heavy Higgs boson via two B jets plus diphoton. Phys. Rev. D 89(11), 115006 (2014). arXiv: 1312.7212

79. A. Papaefstathiou, Discovering Higgs boson pair production through rare final states at a $100 \mathrm{TeV}$ collider. Phys. Rev. D 91(11), 113016 (2015). arXiv:1504.04621
80. S. von Buddenbrock, N. Chakrabarty, A.S. Cornell, D. Kar, M. Kumar, T. Mandal, B. Mellado, B. Mukhopadhyaya, R.G. Reed, The compatibility of LHC run 1 data with a heavy scalar of mass around $270 \mathrm{GeV}$ (2015). arXiv: 1506.00612

81. Q.-H. Cao, B. Yan, D.-M. Zhang, H. Zhang, Resolving the degeneracy in single Higgs production with Higgs pair production. Phys. Lett. B 752, 285-290 (2016). arXiv: 1508.06512

82. Q.-H. Cao, Y. Liu, B. Yan, Measuring trilinear Higgs coupling in $W H H$ and $Z H H$ productions at the HL-LHC. Phys. Rev. D95(7) 073006 (2017). arXiv:1511.03311

83. F.P. Huang, P.-H. Gu, P.-F. Yin, Z.-H. Yu, X. Zhang, Testing the electroweak phase transition and electroweak baryogenesis at the LHC and a circular electron-positron collider. Phys. Rev. D 93(10), 103515 (2016). arXiv:1511.03969

84. J.K. Behr, D. Bortoletto, J.A. Frost, N.P. Hartland, C. Issever, J. Rojo, Boosting Higgs pair production in the $b \bar{b} b \bar{b}$ final state with multivariate techniques. Eur. Phys. J. C 76(7), 386 (2016). arXiv: 1512.08928

85. J. Cao, L. Shang, W. Su, Y. Zhang, J. Zhu, Interpreting the 750 $\mathrm{GeV}$ diphoton excess in the minimal dilaton model. Eur. Phys. J. C 76(5), 239 (2016). arXiv: 1601.02570

86. Z. Kang, Bound states via Higgs exchanging and resonant diHiggs (2016). arXiv:1606.01531

87. S. von Buddenbrock, N. Chakrabarty, A.S. Cornell, D. Kar, M. Kumar, T. Mandal, B. Mellado, B. Mukhopadhyaya, R.G. Reed, X. Ruan, Phenomenological signatures of additional scalar bosons at the LHC. Eur. Phys. J. C 76(10), 580 (2016). arXiv:1606.01674

88. S. Fichet, G. von Gersdorff, E. Pontón, R. Rosenfeld, The excitation of the global symmetry-breaking vacuum in composite Higgs models. JHEP 09, 158 (2016). arXiv:1607.03125

89. S. Fichet, G. von Gersdorff, E. Pontón, R. Rosenfeld, The global Higgs as a signal for compositeness at the LHC. JHEP 01, 012 (2017). arXiv: 1608.01995

90. T. Huang, J.M. No, L. Pernié, M. Ramsey-Musolf, A. Safonov, M. Spannowsky, P. Winslow, Resonant di-Higgs production in the $b \bar{b} W W$ channel: probing the electroweak phase transition at the LHC (2017). arXiv: 1701.04442

91. T. Plehn, M. Rauch, The quartic Higgs coupling at hadron colliders. Phys. Rev. D 72, 053008 (2005). arXiv:hep-ph/0507321

92. F. Maltoni, E. Vryonidou, M. Zaro, Top-quark mass effects in double and triple Higgs production in gluon-gluon fusion at NLO. JHEP 11, 079 (2014). arXiv: 1408.6542

93. A. Papaefstathiou, K. Sakurai, Triple Higgs boson production at a $100 \mathrm{TeV}$ proton-proton collider. JHEP 02, 006 (2016). arXiv: 1508.06524

94. M. Bauer, M. Neubert, Minimal leptoquark explanation for the $\mathrm{R}_{D^{(*)}}, R_{K}$, and $(g-2)_{g}$ anomalies. Phys. Rev. Lett. 116(14), 141802 (2016). arXiv: 1511.01900

95. A. Djouadi, The anatomy of electro-weak symmetry breaking. I: The Higgs boson in the standard model. Phys. Rep. 457, 1-216 (2008). arXiv:hep-ph/0503172

96. T. Abe, R. Kitano, Y. Konishi, K.-Y. Oda, J. Sato, S. Sugiyama, Minimal dilaton model. Phys. Rev. D 86, 115016 (2012). arXiv: 1209.4544

97. M. Carena, I. Low, C.E.M. Wagner, Implications of a modified Higgs to diphoton decay width. JHEP 08, 060 (2012). arXiv: 1206.1082

98. H.M. Lee, D. Kim, K. Kong, S.C. Park, Diboson excesses demystified in effective field theory approach. JHEP 11, 150 (2015). arXiv: 1507.06312

99. P.M. Nadolsky, H.-L. Lai, Q.-H. Cao, J. Huston, J. Pumplin, D. Stump, W.-K. Tung, C.P. Yuan, Implications of CTEQ global analysis for collider observables. Phys. Rev. D 78, 013004 (2008). arXiv:0802.0007

100. FCC website, http://cern.ch/fcc 
101. M. Benedikt, D. Schulte, J. Wenninger, F. Zimmermann, Challenges for highest energy circular colliders. No. CERN-ACC2014-0153 (2014)

102. A. Ball et al., Future circular collider study hadron collider parameters. EDMS No. 1342402 (2014)

103. ATLAS, CMS, G. Aad et al., Measurements of the Higgs boson production and decay rates and constraints on its couplings from a combined ATLAS and CMS analysis of the LHC pp collision data at $\sqrt{s}=7$ and 8 TeV. JHEP 08, 045 (2016). arXiv:1606.02266

104. ATLAS Collaboration, Study of the Higgs boson properties and search for high-mass scalar resonances in the $H \rightarrow Z Z^{*} \rightarrow 4 \ell$ decay channel at $\sqrt{s}=13 \mathrm{TeV}$ with the ATLAS detector. Technical Report ATLAS-CONF-2016-079, CERN, Geneva (2016)

105. ATLAS Collaboration, Search for scalar diphoton resonances with $15.4 \mathrm{fb}^{-1}$ of data collected at $\sqrt{s}=13 \mathrm{TeV}$ in 2015 and 2016 with the ATLAS detector. Technical Report ATLAS-CONF-2016-059, CERN, Geneva (2016)

106. ATLAS, G. Aad et al., Search for production of vector-like quark pairs and of four top quarks in the lepton-plus-jets final state in $p p$ collisions at $\sqrt{s}=8 \mathrm{TeV}$ with the ATLAS detector. JHEP 08, 105 (2015). arXiv: 1505.04306

107. ATLAS Collaboration, Search for pair production of vector-like top partners in events with exactly one lepton and large missing transverse momentum in $\sqrt{s}=13 \mathrm{TeV} p p$ collisions with the ATLAS detector. Technical Report ATLAS-CONF-2016-101, CERN, Geneva (2016)
108. ATLAS, M. Aaboud et al., Search for scalar leptoquarks in pp collisions at $\sqrt{s}=13 \mathrm{TeV}$ with the ATLAS experiment. New J. Phys. 18(9), 093016 (2016). arXiv: 1605.06035

109. CMS, V. Khachatryan et al., Search for heavy neutrinos or thirdgeneration leptoquarks in final states with two hadronically decaying tau leptons and two jets in proton-proton collisions at $\sqrt{s}=$ 13 TeV. JHEP 077, 1703 (2017). arXiv:1612.01190

110. R.S. Chivukula, P. Ittisamai, K. Mohan, E.H. Simmons, Color discriminant variable and scalar diquarks at the LHC. Phys. Rev. D 92(7), 075020 (2015). arXiv:1507.06676

111. CMS, A.M. Sirunyan et al., Search for dijet resonances in proton-proton collisions at $\sqrt{s}=13 \mathrm{TeV}$ and constraints on dark matter and other models. Phys. Lett. B (2016) (submitted). arXiv: 1611.03568

112. G. Ortona, Search for double Higgs production or decay using the CMS detector. Talk presented at ICHEP 2016. Chicago, 3-10 August 2016

113. T. Varol, Search for di-Higgs production with the atlas detector. Talk presented at ICHEP 2016. Chicago, 3-10 August 2016

114. Y. Hamada, H. Kawai, K.-Y. Oda, Bare Higgs mass at Planck scale. Phys. Rev. D 87(5), 053009 (2013). arXiv:1210.2538 [Erratum: Phys. Rev. D 89(5), 059901 (2014)]

115. D. Buttazzo, G. Degrassi, P.P. Giardino, G.F. Giudice, F. Sala, A. Salvio, A. Strumia, Investigating the near-criticality of the Higgs boson. JHEP 12, 089 (2013). arXiv:1307.3536 